Atomic data for radiative transitions in the third spectra of rhodium (Rh III), palladium (Pd III) and silver (Ag III)

This content has been downloaded from IOPscience. Please scroll down to see the full text. 2013 Phys. Scr. 88065302

(http://iopscience.iop.org/1402-4896/88/6/065302)

View the table of contents for this issue, or go to the journal homepage for more

Download details:

IP Address: 193.190.193.1

This content was downloaded on 14/11/2013 at 10:05

Please note that terms and conditions apply. 


\title{
Atomic data for radiative transitions in the third spectra of rhodium (Rh III), palladium (Pd III) and silver (Ag III)
}

\author{
W Zhang ${ }^{1}$, P Palmeri ${ }^{1}$ and P Quinet ${ }^{1,2}$ \\ ${ }^{1}$ Astrophysique et Spectroscopie, Université de Mons, B-7000 Mons, Belgium \\ 2 IPNAS, Université de Liège, B15 Sart Tilman, B-4000 Liège, Belgium \\ E-mail: quinet@umons.ac.be
}

Received 25 April 2013

Accepted for publication 14 October 2013

Published 13 November 2013

Online at stacks.iop.org/PhysScr/88/065302

\begin{abstract}
A new set of radiative decay rates in doubly ionized rhodium ( $\mathrm{Rh}$ III), palladium (Pd III) and silver (Ag III) has been computed by means of the pseudo-relativistic Hartree-Fock method including core-polarization effects (HFR+CPOL) and semi-empirical optimization of radial energy parameters. For these three ions, oscillator strengths and transition probabilities for spectral lines appearing in the ultraviolet region from 70 to $250 \mathrm{~nm}$ are reported for the first time. The accuracy of these results has been estimated through the excellent agreement observed between experimental lifetime measurements and similar HFR+CPOL calculations performed recently in isoelectronic ions Ru II and Rh II.
\end{abstract}

S Online supplementary data available from stacks.iop.org/PhysScr/88/065302/mmedia

\section{Introduction}

The calculation of electronic structure and radiative parameters for atoms and lowly charged ions belonging to the fifth row of the periodic table (from $\mathrm{Rb}$ to $\mathrm{Xe}$ ) is a demanding and time-consuming challenge. If neutral and singly ionized atoms of this group have been the subject of many different studies on both experimental and theoretical sides, doubly ionized species considerably suffer from the lack of accurate atomic data. The present paper focuses on the particular cases of Rh III, Pd III and Ag III for which no radiative rates have been published so far. These three ions are characterized by similar $4 \mathrm{~d}^{k}$ ground-state configurations with $k=7,8$ and 9 for Rh III, Pd III and Ag III, respectively.

In astrophysics, rhodium, palladium and silver are among the elements important for testing current nucleosynthesis models (Mashonkina 2009) for which a detailed analysis of the rapid (r-) and slow (s-) neutron capture processes is not possible without reliable atomic data. As an example, a recent spectral synthesis of 71 stars by Hansen et al (2012) showed clear indications that a second/weak r-process is responsible for the formation of $\mathrm{Pd}$ and $\mathrm{Ag}$. On the basis of the comparison to model predictions, these authors found that the conditions under which this process takes place differ from those for the main r-process in needing lower neutron number densities, lower neutron-to-seed ratios, and lower entropies and/or higher electron abundances. The understanding of the large overabundances of some heavy elements in chemically peculiar stars requires also a large number of radiative parameters for neutral, singly and doubly ionized heavy atoms (see e.g. Wallerstein et al 1997, Jorissen 2004).

In the present paper, we report on calculations of oscillator strengths and transition probabilities in Rh III, Pd III and Ag III performed using the relativistic Hartree-Fock approach including core-polarization effects. This work is an extension of our recent investigations of the fifth row elements Y II, Y III (Biémont et al 2011), Zr II (Malcheva et al 2006), Nb I (Malcheva et al 2011), Nb II, Nb III (Nilsson et al 2010), Mo II (Quinet 2002, Lundberg et al 2010, Jiang et al 2012), Tc II (Palmeri et al 2007), Ru I (Fivet et al 2009), Ru II, Ru III (Palmeri et al 2009), Rh II (Quinet et al 2011, 2012), Pd I (Xu et al 2006), Ag II (Biémont et al 2005, Campos et al 2005), Sn I (Zhang et al 2008, 2009, 2010), Sb I (Hartman et al 2010), Te II and Te III (Zhang et al 2013).

The excellent agreement observed for the isoelectronic ions Ru II and Rh II when using similar pseudo-relativistic Hartree-Fock method including core-polarization effects (HFR+CPOL) models allows the assessment of the reliability 
Table 4. Oscillator strengths and transition probabilities in Rh III $(\log g f>-0.5)$.

\begin{tabular}{|c|c|c|c|c|c|c|c|c|}
\hline \multirow{2}{*}{$\begin{array}{l}\text { Wavelength }^{\mathrm{a}} \\
(\mathrm{nm})\end{array}$} & \multicolumn{3}{|c|}{ Lower level $^{\mathrm{b}}$} & \multicolumn{3}{|c|}{ Upper level $^{\mathrm{b}}$} & \multirow[b]{2}{*}{$\log g f^{\mathrm{c}}$} & \multirow{2}{*}{$\begin{array}{l}g A^{\mathrm{c}} \\
\left(\mathrm{s}^{-1}\right)\end{array}$} \\
\hline & $E\left(\mathrm{~cm}^{-1}\right)$ & Parity & $J$ & $E\left(\mathrm{~cm}^{-1}\right)$ & Parity & $J$ & & \\
\hline 85.228 & 2148 & (e) & $7 / 2$ & 119481 & (o) & $7 / 2$ & -0.48 & $3.01 \times 10^{9}$ \\
\hline 85.477 & 0 & (e) & $9 / 2$ & 116991 & (o) & $9 / 2$ & -0.36 & $3.98 \times 10^{9}$ \\
\hline 85.988 & 0 & (e) & $9 / 2$ & 116296 & (o) & $9 / 2$ & 0.02 & $9.55 \times 10^{9}$ \\
\hline 86.133 & 3486 & (e) & $5 / 2$ & 119586 & (o) & $5 / 2$ & -0.34 & $4.15 \times 10^{9}$ \\
\hline 86.200 & 15130 & (e) & $7 / 2$ & 131138 & (o) & $5 / 2$ & -0.42 & $3.40 \times 10^{9}$ \\
\hline 86.211 & 3486 & (e) & $5 / 2$ & 119481 & (o) & $7 / 2$ & -0.28 & $4.73 \times 10^{9}$ \\
\hline 86.376 & 2148 & (e) & $7 / 2$ & 117921 & (o) & $7 / 2$ & -0.15 & $6.29 \times 10^{9}$ \\
\hline 86.622 & 0 & (e) & $9 / 2$ & 115445 & (o) & $11 / 2$ & -0.47 & $3.04 \times 10^{9}$ \\
\hline 86.758 & 4322 & (e) & $3 / 2$ & 119586 & (o) & $5 / 2$ & -0.44 & $3.18 \times 10^{9}$ \\
\hline 87.038 & 13030 & (e) & $9 / 2$ & 127923 & (o) & $7 / 2$ & 0.27 & $1.64 \times 10^{10}$ \\
\hline 87.076 & 2148 & (e) & $7 / 2$ & 116991 & (o) & $9 / 2$ & -0.11 & $6.85 \times 10^{9}$ \\
\hline 87.425 & 3486 & (e) & $5 / 2$ & 117870 & (o) & $5 / 2$ & -0.44 & $3.14 \times 10^{9}$ \\
\hline 87.786 & 13030 & (e) & $9 / 2$ & 126944 & (o) & $9 / 2$ & -0.20 & $5.51 \times 10^{9}$ \\
\hline 87.839 & 2148 & (e) & $7 / 2$ & 115992 & (o) & $5 / 2$ & -0.33 & $4.06 \times 10^{9}$ \\
\hline 89.432 & 0 & (e) & $9 / 2$ & 111817 & (o) & $9 / 2$ & -0.25 & $4.65 \times 10^{9}$ \\
\hline 89.658 & 19576 & (e) & $9 / 2$ & 131111 & (o) & $7 / 2$ & 0.03 & $8.84 \times 10^{9}$ \\
\hline 89.755 & 0 & (e) & $9 / 2$ & 111414 & (o) & $11 / 2$ & 0.06 & $9.55 \times 10^{9}$ \\
\hline 89.765 & 15130 & (e) & $7 / 2$ & 126532 & (o) & $7 / 2$ & -0.08 & $6.89 \times 10^{9}$ \\
\hline 90.020 & 13030 & (e) & $9 / 2$ & 124116 & (o) & $11 / 2$ & -0.29 & $4.23 \times 10^{9}$ \\
\hline 90.022 & 15130 & (e) & $7 / 2$ & 126213 & (o) & $7 / 2$ & -0.49 & $2.68 \times 10^{9}$ \\
\hline 90.057 & 0 & (e) & $9 / 2$ & 111041 & (o) & $11 / 2$ & -0.33 & $3.89 \times 10^{9}$ \\
\hline 90.976 & 13030 & (e) & $9 / 2$ & 122949 & (o) & $9 / 2$ & 0.04 & $8.76 \times 10^{9}$ \\
\hline 91.123 & 15130 & (e) & $7 / 2$ & 124871 & (o) & $7 / 2$ & -0.32 & $3.89 \times 10^{9}$ \\
\hline 91.183 & 2148 & (e) & $7 / 2$ & 111817 & (o) & $9 / 2$ & -0.45 & $2.82 \times 10^{9}$ \\
\hline 91.375 & 21699 & (e) & $3 / 2$ & 131138 & (o) & $5 / 2$ & -0.35 & $3.55 \times 10^{9}$ \\
\hline 91.499 & 13030 & (e) & $9 / 2$ & 122321 & (o) & $7 / 2$ & -0.14 & $5.79 \times 10^{9}$ \\
\hline 91.982 & 21699 & (e) & $3 / 2$ & 130416 & (o) & $1 / 2$ & -0.29 & $4.04 \times 10^{9}$ \\
\hline 92.008 & 15130 & (e) & $7 / 2$ & 123815 & (o) & $9 / 2$ & -0.18 & $5.22 \times 10^{9}$ \\
\hline 92.574 & 19576 & (e) & $9 / 2$ & 127598 & (o) & $9 / 2$ & 0.46 & $2.22 \times 10^{10}$ \\
\hline 92.677 & 17425 & (e) & $11 / 2$ & 125326 & (o) & $9 / 2$ & -0.05 & $7.00 \times 10^{9}$ \\
\hline 93.129 & 17425 & (e) & $11 / 2$ & 124802 & (o) & $9 / 2$ & -0.48 & $2.57 \times 10^{9}$ \\
\hline 93.728 & 17425 & (e) & $11 / 2$ & 124116 & (o) & $11 / 2$ & 0.42 & $1.99 \times 10^{10}$ \\
\hline 93.743 & 13030 & (e) & $9 / 2$ & 119705 & (o) & $11 / 2$ & -0.21 & $4.68 \times 10^{9}$ \\
\hline 93.858 & 18277 & (e) & $5 / 2$ & 124821 & (o) & $3 / 2$ & -0.49 & $2.43 \times 10^{9}$ \\
\hline 93.993 & 17425 & (e) & $11 / 2$ & 123815 & (o) & $9 / 2$ & -0.41 & $2.95 \times 10^{9}$ \\
\hline 95.336 & 17425 & (e) & $11 / 2$ & 122317 & (o) & $11 / 2$ & -0.39 & $3.01 \times 10^{9}$ \\
\hline 95.472 & 13030 & (e) & $9 / 2$ & & (o) & $9 / 2$ & -0.26 & $4.01 \times 10^{9}$ \\
\hline 95.843 & 26801 & (e) & $5 / 2$ & 131138 & (o) & $5 / 2$ & -0.44 & $2.64 \times 10^{9}$ \\
\hline 96.113 & 18277 & (e) & $5 / 2$ & 122321 & (o) & $7 / 2$ & -0.32 & $3.45 \times 10^{9}$ \\
\hline 99.161 & 0 & (e) & $9 / 2$ & 100846 & (o) & $7 / 2$ & -0.05 & $6.01 \times 10^{9}$ \\
\hline 99.248 & 0 & (e) & $9 / 2$ & 100757 & (o) & $9 / 2$ & -0.36 & $2.93 \times 10^{9}$ \\
\hline 100.291 & 2148 & (e) & $7 / 2$ & 101858 & (o) & $5 / 2$ & -0.27 & $3.56 \times 10^{9}$ \\
\hline 100.960 & 3486 & (e) & $5 / 2$ & 102535 & (o) & $3 / 2$ & -0.38 & $2.76 \times 10^{9}$ \\
\hline 102.631 & 27890 & (e) & $7 / 2$ & 125326 & (o) & $9 / 2$ & -0.19 & $4.12 \times 10^{9}$ \\
\hline 168.857 & 66104 & (e) & $11 / 2$ & 125326 & (o) & $9 / 2$ & -0.47 & $8.02 \times 10^{8}$ \\
\hline 172.940 & 43022 & (e) & $9 / 2$ & 100846 & (o) & $7 / 2$ & -0.37 & $9.51 \times 10^{8}$ \\
\hline 175.152 & 62353 & (e) & $13 / 2$ & 119446 & (o) & $13 / 2$ & -0.17 & $1.48 \times 10^{9}$ \\
\hline 176.743 & 45278 & (e) & $5 / 2$ & 101858 & (o) & $5 / 2$ & -0.46 & $7.41 \times 10^{8}$ \\
\hline 176.842 & 44394 & (e) & $7 / 2$ & 100942 & (o) & $5 / 2$ & -0.30 & $1.05 \times 10^{9}$ \\
\hline 177.245 & 68019 & (e) & $7 / 2$ & 124439 & (o) & $5 / 2$ & -0.20 & $1.32 \times 10^{9}$ \\
\hline 177.315 & 45877 & (e) & $3 / 2$ & 102273 & (o) & $3 / 2$ & -0.39 & $8.59 \times 10^{8}$ \\
\hline 177.398 & 74741 & (e) & $7 / 2$ & 131111 & (o) & $7 / 2$ & -0.44 & $7.79 \times 10^{8}$ \\
\hline 177.421 & 44394 & (e) & $7 / 2$ & 100757 & (o) & $9 / 2$ & -0.15 & $1.51 \times 10^{9}$ \\
\hline 177.987 & 62353 & (e) & $13 / 2$ & 118536 & (o) & $11 / 2$ & -0.24 & $1.20 \times 10^{9}$ \\
\hline 178.424 & 46227 & (e) & $1 / 2$ & 102273 & (o) & $3 / 2$ & -0.16 & $1.45 \times 10^{9}$ \\
\hline 178.459 & 67775 & (e) & $5 / 2$ & 123810 & (o) & $3 / 2$ & -0.28 & $1.09 \times 10^{9}$ \\
\hline 178.494 & 43022 & (e) & $9 / 2$ & 99046 & (o) & $7 / 2$ & -0.15 & $1.48 \times 10^{9}$ \\
\hline 179.650 & 45278 & (e) & $5 / 2$ & 100942 & (o) & $5 / 2$ & -0.34 & $9.32 \times 10^{8}$ \\
\hline 181.602 & 45877 & (e) & $3 / 2$ & 100942 & (o) & $5 / 2$ & -0.04 & $1.84 \times 10^{9}$ \\
\hline 181.818 & 62870 & (e) & $7 / 2$ & 117870 & (o) & $5 / 2$ & -0.05 & $1.78 \times 10^{9}$ \\
\hline 182.209 & 64222 & (e) & $9 / 2$ & 119103 & (o) & $9 / 2$ & -0.33 & $9.42 \times 10^{8}$ \\
\hline 182.544 & 71432 & (e) & $7 / 2$ & 126213 & (o) & $7 / 2$ & -0.21 & $1.24 \times 10^{9}$ \\
\hline 182.656 & 64733 & (e) & $7 / 2$ & 119481 & (o) & $7 / 2$ & -0.45 & $7.05 \times 10^{8}$ \\
\hline 182.737 & 67594 & (e) & $9 / 2$ & 122317 & (o) & $11 / 2$ & -0.47 & $6.73 \times 10^{8}$ \\
\hline
\end{tabular}


Table 4. (Continued.)

\begin{tabular}{|c|c|c|c|c|c|c|c|c|}
\hline \multirow{2}{*}{$\begin{array}{l}\text { Wavelength } \\
(\mathrm{nm})\end{array}$} & \multicolumn{3}{|c|}{ Lower level $^{\text {b }}$} & \multicolumn{3}{|c|}{ Upper level $^{\mathrm{b}}$} & \multirow[b]{2}{*}{$\log g f^{\mathrm{c}}$} & \multirow{2}{*}{$\begin{array}{l}g A^{\mathrm{c}} \\
\left(\mathrm{s}^{-1}\right)\end{array}$} \\
\hline & $E\left(\mathrm{~cm}^{-1}\right)$ & Parity & $J$ & $E\left(\mathrm{~cm}^{-1}\right)$ & Parity & $J$ & & \\
\hline 183.205 & 43022 & (e) & $9 / 2$ & 97606 & (o) & $11 / 2$ & 0.67 & $9.34 \times 10^{9}$ \\
\hline 183.298 & 76583 & (e) & $7 / 2$ & 131138 & (o) & $5 / 2$ & 0.13 & $2.70 \times 10^{9}$ \\
\hline 183.842 & 69722 & (e) & $11 / 2$ & 124116 & (o) & $11 / 2$ & -0.43 & $7.35 \times 10^{8}$ \\
\hline 183.974 & 62402 & (e) & $9 / 2$ & 116758 & (o) & $11 / 2$ & -0.21 & $1.21 \times 10^{9}$ \\
\hline 184.112 & 64222 & (e) & $9 / 2$ & 118536 & (o) & $11 / 2$ & -0.34 & $9.01 \times 10^{8}$ \\
\hline 184.427 & 62536 & (e) & $11 / 2$ & 116758 & (o) & $11 / 2$ & -0.14 & $1.43 \times 10^{9}$ \\
\hline 184.864 & 69722 & (e) & $11 / 2$ & 123815 & (o) & $9 / 2$ & -0.41 & $7.58 \times 10^{8}$ \\
\hline 185.549 & 71432 & (e) & $7 / 2$ & 125326 & (o) & $9 / 2$ & -0.28 & $1.01 \times 10^{9}$ \\
\hline 185.551 & 62402 & (e) & $9 / 2$ & 116296 & (o) & $9 / 2$ & -0.11 & $1.53 \times 10^{9}$ \\
\hline 185.984 & 45278 & (e) & $5 / 2$ & 99046 & (o) & $7 / 2$ & 0.24 & $3.34 \times 10^{9}$ \\
\hline 186.078 & 70946 & (e) & $5 / 2$ & 124687 & (o) & $5 / 2$ & -0.24 & $1.11 \times 10^{9}$ \\
\hline 186.941 & 70946 & (e) & $5 / 2$ & 124439 & (o) & $5 / 2$ & -0.34 & $8.58 \times 10^{8}$ \\
\hline 187.130 & 71432 & (e) & $7 / 2$ & 124871 & (o) & $7 / 2$ & -0.17 & $1.28 \times 10^{9}$ \\
\hline 187.131 & 69747 & (e) & $9 / 2$ & 123185 & (o) & $7 / 2$ & -0.48 & $6.32 \times 10^{8}$ \\
\hline 187.167 & 71259 & (e) & $3 / 2$ & 124687 & (o) & $5 / 2$ & -0.09 & $1.54 \times 10^{9}$ \\
\hline 187.175 & 62870 & (e) & $7 / 2$ & 116296 & (o) & $9 / 2$ & -0.47 & $6.46 \times 10^{8}$ \\
\hline 187.287 & 77744 & (e) & $3 / 2$ & 131138 & (o) & $5 / 2$ & -0.47 & $6.41 \times 10^{8}$ \\
\hline 187.365 & 64874 & (e) & $5 / 2$ & 118246 & (o) & $7 / 2$ & -0.27 & $1.03 \times 10^{9}$ \\
\hline 187.370 & 71432 & (e) & $7 / 2$ & 124802 & (o) & $9 / 2$ & 0.30 & $3.74 \times 10^{9}$ \\
\hline 187.471 & 66104 & (e) & $11 / 2$ & 119446 & (o) & $13 / 2$ & 0.46 & $5.55 \times 10^{9}$ \\
\hline 187.873 & 74371 & (e) & $11 / 2$ & 127598 & (o) & $9 / 2$ & 0.25 & $3.40 \times 10^{9}$ \\
\hline 187.963 & 69747 & (e) & $9 / 2$ & 122949 & (o) & $9 / 2$ & 0.07 & $2.19 \times 10^{9}$ \\
\hline 188.066 & 44394 & (e) & $7 / 2$ & 97567 & (o) & $9 / 2$ & 0.48 & $5.70 \times 10^{9}$ \\
\hline 188.212 & 62353 & (e) & $13 / 2$ & 115484 & (o) & $13 / 2$ & -0.40 & $7.46 \times 10^{8}$ \\
\hline 188.492 & 64222 & (e) & $9 / 2$ & 117274 & (o) & $11 / 2$ & 0.09 & $2.30 \times 10^{9}$ \\
\hline 188.528 & 62402 & (e) & $9 / 2$ & 115445 & (o) & $11 / 2$ & 0.16 & $2.70 \times 10^{9}$ \\
\hline 188.537 & 64733 & (e) & $7 / 2$ & 117773 & (o) & $9 / 2$ & -0.25 & $1.07 \times 10^{9}$ \\
\hline 188.736 & 44394 & (e) & $7 / 2$ & 97378 & (o) & $7 / 2$ & 0.09 & $2.31 \times 10^{9}$ \\
\hline 188.863 & 62536 & (e) & $11 / 2$ & 115484 & (o) & $13 / 2$ & 0.52 & $6.25 \times 10^{9}$ \\
\hline 190.131 & 69722 & (e) & $11 / 2$ & 122317 & (o) & $11 / 2$ & 0.37 & $4.25 \times 10^{9}$ \\
\hline 190.152 & 65280 & (e) & $3 / 2$ & 117870 & (o) & $5 / 2$ & -0.18 & $1.20 \times 10^{9}$ \\
\hline 190.431 & 70673 & (e) & $7 / 2$ & 123185 & (o) & $7 / 2$ & -0.12 & $1.39 \times 10^{9}$ \\
\hline 190.722 & 66104 & (e) & $11 / 2$ & 118536 & (o) & $11 / 2$ & 0.03 & $1.96 \times 10^{9}$ \\
\hline 191.015 & 45278 & (e) & $5 / 2$ & 97630 & (o) & $5 / 2$ & -0.03 & $1.70 \times 10^{9}$ \\
\hline 191.207 & 62353 & (e) & $13 / 2$ & 114652 & (o) & $13 / 2$ & 0.19 & $2.83 \times 10^{9}$ \\
\hline 191.897 & 67594 & (e) & $9 / 2$ & 119705 & (o) & $11 / 2$ & 0.41 & $4.64 \times 10^{9}$ \\
\hline 191.938 & 45278 & (e) & $5 / 2$ & 97378 & (o) & $7 / 2$ & -0.10 & $1.43 \times 10^{9}$ \\
\hline 191.962 & 64874 & (e) & $5 / 2$ & 116968 & (o) & $7 / 2$ & -0.29 & $9.33 \times 10^{8}$ \\
\hline 192.237 & 67775 & (e) & $5 / 2$ & 119794 & (o) & $7 / 2$ & -0.17 & $1.22 \times 10^{9}$ \\
\hline 192.444 & 62402 & (e) & $9 / 2$ & 114365 & (o) & $11 / 2$ & -0.07 & $1.52 \times 10^{9}$ \\
\hline 192.562 & 62870 & (e) & $7 / 2$ & 114801 & (o) & $9 / 2$ & -0.43 & $6.73 \times 10^{8}$ \\
\hline 192.707 & 45877 & (e) & $3 / 2$ & 97769 & (o) & $3 / 2$ & -0.12 & $1.38 \times 10^{9}$ \\
\hline 192.726 & 67594 & (e) & $9 / 2$ & 119481 & (o) & $7 / 2$ & -0.11 & $1.40 \times 10^{9}$ \\
\hline 192.940 & 62536 & (e) & $11 / 2$ & 114365 & (o) & $11 / 2$ & -0.11 & $1.41 \times 10^{9}$ \\
\hline 193.039 & 68019 & (e) & $7 / 2$ & 119822 & (o) & $9 / 2$ & 0.40 & $4.54 \times 10^{9}$ \\
\hline 193.179 & 62353 & (e) & $13 / 2$ & 114118 & (o) & $15 / 2$ & 0.79 & $1.10 \times 10^{10}$ \\
\hline 193.224 & 45877 & (e) & $3 / 2$ & 97630 & (o) & $5 / 2$ & -0.04 & $1.63 \times 10^{9}$ \\
\hline 193.402 & 67775 & (e) & $5 / 2$ & 119481 & (o) & $7 / 2$ & -0.13 & $1.31 \times 10^{9}$ \\
\hline 193.445 & 62536 & (e) & $11 / 2$ & 114230 & (o) & $9 / 2$ & -0.15 & $1.25 \times 10^{9}$ \\
\hline 193.453 & 68019 & (e) & $7 / 2$ & 119712 & (o) & $7 / 2$ & -0.08 & $1.47 \times 10^{9}$ \\
\hline 193.541 & 62402 & (e) & $9 / 2$ & 114071 & (o) & $7 / 2$ & -0.22 & $1.06 \times 10^{9}$ \\
\hline 193.846 & 46227 & (e) & $1 / 2$ & 97814 & (o) & $1 / 2$ & -0.26 & $9.91 \times 10^{8}$ \\
\hline 193.926 & 68019 & (e) & $7 / 2$ & 119586 & (o) & $5 / 2$ & -0.17 & $1.20 \times 10^{9}$ \\
\hline 193.939 & 64733 & (e) & $7 / 2$ & 116296 & (o) & $9 / 2$ & 0.08 & $2.16 \times 10^{9}$ \\
\hline 194.017 & 46227 & (e) & $1 / 2$ & 97769 & (o) & $3 / 2$ & -0.33 & $8.37 \times 10^{8}$ \\
\hline 194.027 & 67775 & (e) & $5 / 2$ & 119314 & (o) & $5 / 2$ & -0.36 & $7.72 \times 10^{8}$ \\
\hline 194.130 & 73814 & (e) & $9 / 2$ & 125326 & (o) & $9 / 2$ & 0.27 & $3.28 \times 10^{9}$ \\
\hline 194.137 & 67594 & (e) & $9 / 2$ & 119103 & (o) & $9 / 2$ & 0.24 & $3.08 \times 10^{9}$ \\
\hline 194.279 & 74741 & (e) & $7 / 2$ & 126213 & (o) & $7 / 2$ & -0.10 & $1.41 \times 10^{9}$ \\
\hline 195.086 & 64733 & (e) & $7 / 2$ & 115992 & (o) & $5 / 2$ & -0.30 & $8.79 \times 10^{8}$ \\
\hline 195.224 & 64222 & (e) & $9 / 2$ & 115445 & (o) & $11 / 2$ & -0.43 & $6.55 \times 10^{8}$ \\
\hline 195.426 & 66104 & (e) & $11 / 2$ & 117274 & (o) & $11 / 2$ & 0.31 & $3.57 \times 10^{9}$ \\
\hline 195.495 & 74741 & (e) & $7 / 2$ & 125893 & (o) & $5 / 2$ & -0.27 & $9.46 \times 10^{8}$ \\
\hline 195.624 & 64874 & (e) & $5 / 2$ & 115992 & (o) & $5 / 2$ & -0.38 & $7.19 \times 10^{8}$ \\
\hline
\end{tabular}


Table 4. (Continued.)

\begin{tabular}{|c|c|c|c|c|c|c|c|c|}
\hline \multirow{2}{*}{$\begin{array}{l}\text { Wavelength } \\
(\mathrm{nm})\end{array}$} & \multicolumn{3}{|c|}{ Lower level $^{\text {b }}$} & \multicolumn{3}{|c|}{ Upper level $^{\mathrm{b}}$} & \multirow[b]{2}{*}{$\log g f^{\mathrm{c}}$} & \multirow{2}{*}{$\begin{array}{l}g A^{\mathrm{c}} \\
\left(\mathrm{s}^{-1}\right)\end{array}$} \\
\hline & $E\left(\mathrm{~cm}^{-1}\right)$ & Parity & $J$ & $E\left(\mathrm{~cm}^{-1}\right)$ & Parity & $J$ & & \\
\hline 196.124 & 73814 & (e) & $9 / 2$ & 124802 & (o) & $9 / 2$ & -0.24 & $9.89 \times 10^{8}$ \\
\hline 196.298 & 67594 & (e) & $9 / 2$ & 118536 & (o) & $11 / 2$ & -0.10 & $1.36 \times 10^{9}$ \\
\hline 196.516 & 66104 & (e) & $11 / 2$ & 116991 & (o) & $9 / 2$ & 0.04 & $1.91 \times 10^{9}$ \\
\hline 196.916 & 70622 & (e) & $3 / 2$ & 121405 & (o) & $3 / 2$ & -0.48 & $5.67 \times 10^{8}$ \\
\hline 197.708 & 64222 & (e) & $9 / 2$ & 114801 & (o) & $9 / 2$ & -0.11 & $1.32 \times 10^{9}$ \\
\hline 198.237 & 62870 & (e) & $7 / 2$ & 113314 & (o) & $7 / 2$ & -0.36 & $7.48 \times 10^{8}$ \\
\hline 198.355 & 64733 & (e) & $7 / 2$ & 115148 & (o) & $7 / 2$ & -0.45 & $5.98 \times 10^{8}$ \\
\hline 198.451 & 71432 & (e) & $7 / 2$ & 121822 & (o) & $5 / 2$ & 0.01 & $1.72 \times 10^{9}$ \\
\hline 198.565 & 76583 & (e) & $7 / 2$ & 126944 & (o) & $9 / 2$ & 0.17 & $2.51 \times 10^{9}$ \\
\hline 198.701 & 67594 & (e) & $9 / 2$ & 117921 & (o) & $7 / 2$ & -0.02 & $1.60 \times 10^{9}$ \\
\hline 198.799 & 73814 & (e) & $9 / 2$ & 124116 & (o) & $11 / 2$ & -0.23 & $9.89 \times 10^{8}$ \\
\hline 199.002 & 70673 & (e) & $7 / 2$ & 120924 & (o) & $5 / 2$ & -0.03 & $1.57 \times 10^{9}$ \\
\hline 199.139 & 77706 & (e) & $5 / 2$ & 127923 & (o) & $7 / 2$ & 0.17 & $2.49 \times 10^{9}$ \\
\hline 199.237 & 66104 & (e) & $11 / 2$ & 116296 & (o) & $9 / 2$ & -0.49 & $5.43 \times 10^{8}$ \\
\hline 199.285 & 67594 & (e) & $9 / 2$ & 117773 & (o) & $9 / 2$ & -0.43 & $6.23 \times 10^{8}$ \\
\hline 199.419 & 67775 & (e) & $5 / 2$ & 117921 & (o) & $7 / 2$ & -0.39 & $6.80 \times 10^{8}$ \\
\hline 199.426 & 64222 & (e) & $9 / 2$ & 114365 & (o) & $11 / 2$ & 0.23 & $2.83 \times 10^{9}$ \\
\hline 199.481 & 74741 & (e) & $7 / 2$ & 124871 & (o) & $7 / 2$ & -0.02 & $1.60 \times 10^{9}$ \\
\hline 199.554 & 62870 & (e) & $7 / 2$ & 112982 & (o) & $5 / 2$ & -0.25 & $9.51 \times 10^{8}$ \\
\hline 199.572 & 74009 & (e) & $13 / 2$ & 124116 & (o) & $11 / 2$ & 0.35 & $3.68 \times 10^{9}$ \\
\hline 199.728 & 64733 & (e) & $7 / 2$ & 114801 & (o) & $9 / 2$ & -0.14 & $1.21 \times 10^{9}$ \\
\hline 199.811 & 69747 & (e) & $9 / 2$ & 119794 & (o) & $7 / 2$ & -0.48 & $5.58 \times 10^{8}$ \\
\hline 199.966 & 64222 & (e) & $9 / 2$ & 114230 & (o) & $9 / 2$ & -0.04 & $1.52 \times 10^{9}$ \\
\hline 200.002 & 69722 & (e) & $11 / 2$ & 119705 & (o) & $11 / 2$ & -0.15 & $1.17 \times 10^{9}$ \\
\hline 200.138 & 76583 & (e) & $7 / 2$ & 126532 & (o) & $7 / 2$ & 0.23 & $2.84 \times 10^{9}$ \\
\hline 200.331 & 68019 & (e) & $7 / 2$ & 117921 & (o) & $7 / 2$ & -0.35 & $7.45 \times 10^{8}$ \\
\hline 200.515 & 62870 & (e) & $7 / 2$ & 112725 & (o) & $9 / 2$ & 0.15 & $2.34 \times 10^{9}$ \\
\hline 200.540 & 64222 & (e) & $9 / 2$ & 114071 & (o) & $7 / 2$ & -0.46 & $5.77 \times 10^{8}$ \\
\hline 200.958 & 74371 & (e) & $11 / 2$ & 124116 & (o) & $11 / 2$ & -0.46 & $5.63 \times 10^{8}$ \\
\hline 201.045 & 69722 & (e) & $11 / 2$ & 119446 & (o) & $13 / 2$ & 0.20 & $2.61 \times 10^{9}$ \\
\hline 201.372 & 74009 & (e) & $13 / 2$ & 123652 & (o) & $15 / 2$ & 0.77 & $9.78 \times 10^{9}$ \\
\hline 201.445 & 62402 & (e) & $9 / 2$ & 112028 & (o) & $7 / 2$ & -0.38 & $6.87 \times 10^{8}$ \\
\hline 201.527 & 69747 & (e) & $9 / 2$ & 119352 & (o) & $9 / 2$ & -0.19 & $1.05 \times 10^{9}$ \\
\hline 201.746 & 54632 & (e) & $7 / 2$ & 104183 & (o) & $5 / 2$ & 0.10 & $2.08 \times 10^{9}$ \\
\hline 202.376 & 67594 & (e) & $9 / 2$ & 116991 & (o) & $9 / 2$ & -0.06 & $1.42 \times 10^{9}$ \\
\hline 202.498 & 56126 & (e) & $5 / 2$ & 105493 & (o) & $3 / 2$ & -0.18 & $1.09 \times 10^{9}$ \\
\hline 202.851 & 62536 & (e) & $11 / 2$ & 111817 & (o) & $9 / 2$ & 0.27 & $3.04 \times 10^{9}$ \\
\hline 203.361 & 62870 & (e) & $7 / 2$ & 112028 & (o) & $7 / 2$ & -0.35 & $7.30 \times 10^{8}$ \\
\hline 203.630 & 64222 & (e) & $9 / 2$ & 113314 & (o) & $7 / 2$ & -0.01 & $1.58 \times 10^{9}$ \\
\hline 203.672 & 62353 & (e) & $13 / 2$ & 111435 & (o) & $13 / 2$ & 0.38 & $3.87 \times 10^{9}$ \\
\hline 203.706 & 74741 & (e) & $7 / 2$ & 123815 & (o) & $9 / 2$ & 0.25 & $2.84 \times 10^{9}$ \\
\hline 203.761 & 62353 & (e) & $13 / 2$ & 111414 & (o) & $11 / 2$ & 0.14 & $2.18 \times 10^{9}$ \\
\hline 203.967 & 62402 & (e) & $9 / 2$ & 111414 & (o) & $11 / 2$ & 0.04 & $1.74 \times 10^{9}$ \\
\hline 203.970 & 72394 & (e) & $5 / 2$ & 121405 & (o) & $3 / 2$ & -0.39 & $6.47 \times 10^{8}$ \\
\hline 204.018 & 43022 & (e) & $9 / 2$ & 92022 & (o) & $7 / 2$ & 0.16 & $2.29 \times 10^{9}$ \\
\hline 204.232 & 68019 & (e) & $7 / 2$ & 116968 & (o) & $7 / 2$ & -0.11 & $1.23 \times 10^{9}$ \\
\hline 204.434 & 62536 & (e) & $11 / 2$ & 111435 & (o) & $13 / 2$ & 0.21 & $2.61 \times 10^{9}$ \\
\hline 204.524 & 62536 & (e) & $11 / 2$ & 111414 & (o) & $11 / 2$ & 0.14 & $2.20 \times 10^{9}$ \\
\hline 204.569 & 77706 & (e) & $5 / 2$ & 126574 & (o) & $5 / 2$ & 0.06 & $1.84 \times 10^{9}$ \\
\hline 204.866 & 43022 & (e) & $9 / 2$ & 91819 & (o) & $9 / 2$ & 0.54 & $5.47 \times 10^{9}$ \\
\hline 204.896 & 69747 & (e) & $9 / 2$ & 118536 & (o) & $11 / 2$ & -0.28 & $8.32 \times 10^{8}$ \\
\hline 205.113 & 67775 & (e) & $5 / 2$ & 116513 & (o) & $5 / 2$ & -0.09 & $1.28 \times 10^{9}$ \\
\hline 205.255 & 57531 & (e) & $1 / 2$ & 106236 & (o) & $1 / 2$ & -0.49 & $5.16 \times 10^{8}$ \\
\hline 205.286 & 66104 & (e) & $11 / 2$ & 114801 & (o) & $9 / 2$ & -0.46 & $5.47 \times 10^{8}$ \\
\hline 205.322 & 62353 & (e) & $13 / 2$ & 111041 & (o) & $11 / 2$ & 0.26 & $2.90 \times 10^{9}$ \\
\hline 205.360 & 70673 & (e) & $7 / 2$ & 119352 & (o) & $9 / 2$ & 0.12 & $2.06 \times 10^{9}$ \\
\hline 205.442 & 64874 & (e) & $5 / 2$ & 113534 & (o) & $3 / 2$ & -0.36 & $6.93 \times 10^{8}$ \\
\hline 205.531 & 62402 & (e) & $9 / 2$ & 111041 & (o) & $11 / 2$ & -0.45 & $5.61 \times 10^{8}$ \\
\hline 205.774 & 64733 & (e) & $7 / 2$ & 113314 & (o) & $7 / 2$ & -0.37 & $6.65 \times 10^{8}$ \\
\hline 205.787 & 74371 & (e) & $11 / 2$ & 122949 & (o) & $9 / 2$ & -0.39 & $6.48 \times 10^{8}$ \\
\hline 205.917 & 66104 & (e) & $11 / 2$ & 114652 & (o) & $13 / 2$ & 0.35 & $3.52 \times 10^{9}$ \\
\hline 206.090 & 73814 & (e) & $9 / 2$ & 122321 & (o) & $7 / 2$ & -0.22 & $9.38 \times 10^{8}$ \\
\hline 206.090 & 77706 & (e) & $5 / 2$ & 126213 & (o) & $7 / 2$ & -0.70 & $3.17 \times 10^{8}$ \\
\hline 206.097 & 62536 & (e) & $11 / 2$ & 111041 & (o) & $11 / 2$ & 0.03 & $1.70 \times 10^{9}$ \\
\hline
\end{tabular}


Table 4. (Continued.)

\begin{tabular}{|c|c|c|c|c|c|c|c|c|}
\hline \multirow{2}{*}{$\begin{array}{l}\text { Wavelength } \\
(\mathrm{nm})\end{array}$} & \multicolumn{3}{|c|}{ Lower level $^{\text {b }}$} & \multicolumn{3}{|c|}{ Upper level $^{\mathrm{b}}$} & \multirow[b]{2}{*}{$\log g f^{\mathrm{c}}$} & \multirow{2}{*}{$\begin{array}{l}g A^{\mathrm{c}} \\
\left(\mathrm{s}^{-1}\right)\end{array}$} \\
\hline & $E\left(\mathrm{~cm}^{-1}\right)$ & Parity & $J$ & $E\left(\mathrm{~cm}^{-1}\right)$ & Parity & $J$ & & \\
\hline 206.104 & 64222 & (e) & $9 / 2$ & 112725 & (o) & $9 / 2$ & -0.44 & $5.68 \times 10^{8}$ \\
\hline 206.107 & 73814 & (e) & $9 / 2$ & 122317 & (o) & $11 / 2$ & 0.11 & $2.01 \times 10^{9}$ \\
\hline 206.202 & 57013 & (e) & $3 / 2$ & 105493 & (o) & $3 / 2$ & -0.38 & $6.61 \times 10^{8}$ \\
\hline 206.410 & 44394 & (e) & $7 / 2$ & 92826 & (o) & $5 / 2$ & 0.15 & $2.22 \times 10^{9}$ \\
\hline 207.173 & 65280 & (e) & $3 / 2$ & 113534 & (o) & $3 / 2$ & -0.38 & $6.55 \times 10^{8}$ \\
\hline 207.194 & 64733 & (e) & $7 / 2$ & 112982 & (o) & $5 / 2$ & -0.32 & $7.40 \times 10^{8}$ \\
\hline 207.367 & 74741 & (e) & $7 / 2$ & 122949 & (o) & $9 / 2$ & -0.41 & $5.97 \times 10^{8}$ \\
\hline 207.574 & 62402 & (e) & $9 / 2$ & 110562 & (o) & $9 / 2$ & 0.34 & $3.41 \times 10^{9}$ \\
\hline 207.624 & 77744 & (e) & $3 / 2$ & 125893 & (o) & $5 / 2$ & -0.22 & $9.29 \times 10^{8}$ \\
\hline 207.684 & 45278 & (e) & $5 / 2$ & 93413 & (o) & $3 / 2$ & 0.04 & $1.71 \times 10^{9}$ \\
\hline 207.801 & 64874 & (e) & $5 / 2$ & 112982 & (o) & $5 / 2$ & -0.46 & $5.34 \times 10^{8}$ \\
\hline 207.932 & 77744 & (e) & $3 / 2$ & 125822 & (o) & $3 / 2$ & -0.11 & $1.18 \times 10^{9}$ \\
\hline 208.020 & 56126 & (e) & $5 / 2$ & 104183 & (o) & $5 / 2$ & -0.36 & $6.74 \times 10^{8}$ \\
\hline 208.154 & 69747 & (e) & $9 / 2$ & 117773 & (o) & $9 / 2$ & -0.23 & $9.20 \times 10^{8}$ \\
\hline 208.300 & 64733 & (e) & $7 / 2$ & 112725 & (o) & $9 / 2$ & -0.35 & $6.81 \times 10^{8}$ \\
\hline 208.519 & 83169 & (e) & $5 / 2$ & 131111 & (o) & $7 / 2$ & 0.21 & $2.50 \times 10^{9}$ \\
\hline 208.560 & 45877 & (e) & $3 / 2$ & 93809 & (o) & $1 / 2$ & -0.22 & $9.31 \times 10^{8}$ \\
\hline 209.106 & 62402 & (e) & $9 / 2$ & 110210 & (o) & $7 / 2$ & -0.23 & $9.05 \times 10^{8}$ \\
\hline 209.609 & 62870 & (e) & $7 / 2$ & 110562 & (o) & $9 / 2$ & -0.07 & $1.30 \times 10^{9}$ \\
\hline 209.897 & 44394 & (e) & $7 / 2$ & 92022 & (o) & $7 / 2$ & 0.05 & $1.69 \times 10^{9}$ \\
\hline 210.037 & 64222 & (e) & $9 / 2$ & 111817 & (o) & $9 / 2$ & -0.45 & $5.30 \times 10^{8}$ \\
\hline 210.138 & 70673 & (e) & $7 / 2$ & 118246 & (o) & $7 / 2$ & -0.47 & $5.14 \times 10^{8}$ \\
\hline 210.248 & 45278 & (e) & $5 / 2$ & 92826 & (o) & $5 / 2$ & -0.44 & $5.48 \times 10^{8}$ \\
\hline 210.338 & 69747 & (e) & $9 / 2$ & 117274 & (o) & $11 / 2$ & -0.08 & $1.25 \times 10^{9}$ \\
\hline 210.905 & 72394 & (e) & $5 / 2$ & 119794 & (o) & $7 / 2$ & -0.19 & $9.76 \times 10^{8}$ \\
\hline 211.171 & 62870 & (e) & $7 / 2$ & 110210 & (o) & $7 / 2$ & 0.01 & $1.51 \times 10^{9}$ \\
\hline 211.273 & 72394 & (e) & $5 / 2$ & 119712 & (o) & $7 / 2$ & 0.04 & $1.62 \times 10^{9}$ \\
\hline 211.374 & 64733 & (e) & $7 / 2$ & 112028 & (o) & $7 / 2$ & -0.34 & $6.87 \times 10^{8}$ \\
\hline 211.489 & 69722 & (e) & $11 / 2$ & 116991 & (o) & $9 / 2$ & -0.20 & $9.28 \times 10^{8}$ \\
\hline 211.650 & 76583 & (e) & $7 / 2$ & 123815 & (o) & $9 / 2$ & -0.31 & $7.30 \times 10^{8}$ \\
\hline 211.683 & 54632 & (e) & $7 / 2$ & 101858 & (o) & $5 / 2$ & -0.21 & $9.11 \times 10^{8}$ \\
\hline 211.854 & 56126 & (e) & $5 / 2$ & 103314 & (o) & $5 / 2$ & -0.44 & $5.45 \times 10^{8}$ \\
\hline 212.005 & 64874 & (e) & $5 / 2$ & 112028 & (o) & $7 / 2$ & -0.08 & $1.23 \times 10^{9}$ \\
\hline 212.180 & 77706 & (e) & $5 / 2$ & 124821 & (o) & $3 / 2$ & -0.16 & $1.02 \times 10^{9}$ \\
\hline 212.247 & 70673 & (e) & $7 / 2$ & 117773 & (o) & $9 / 2$ & -0.28 & $7.88 \times 10^{8}$ \\
\hline 212.536 & 69722 & (e) & $11 / 2$ & 116758 & (o) & $11 / 2$ & -0.39 & $6.09 \times 10^{8}$ \\
\hline 212.846 & 57013 & (e) & $3 / 2$ & 103980 & (o) & $3 / 2$ & -0.49 & $4.76 \times 10^{8}$ \\
\hline 212.928 & 45877 & (e) & $3 / 2$ & 92826 & (o) & $5 / 2$ & -0.44 & $5.36 \times 10^{8}$ \\
\hline 213.062 & 72394 & (e) & $5 / 2$ & 119314 & (o) & $5 / 2$ & -0.13 & $1.09 \times 10^{9}$ \\
\hline 213.519 & 64222 & (e) & $9 / 2$ & 111041 & (o) & $11 / 2$ & -0.39 & $6.00 \times 10^{8}$ \\
\hline 213.691 & 68019 & (e) & $7 / 2$ & 114801 & (o) & $9 / 2$ & -0.49 & $4.70 \times 10^{8}$ \\
\hline 213.737 & 67594 & (e) & $9 / 2$ & 114365 & (o) & $11 / 2$ & -0.46 & $5.03 \times 10^{8}$ \\
\hline 214.428 & 66104 & (e) & $11 / 2$ & 112725 & (o) & $9 / 2$ & -0.27 & $7.88 \times 10^{8}$ \\
\hline 215.224 & 57531 & (e) & $1 / 2$ & 103980 & (o) & $3 / 2$ & -0.04 & $1.33 \times 10^{9}$ \\
\hline 215.407 & 56126 & (e) & $5 / 2$ & 102535 & (o) & $3 / 2$ & -0.26 & $7.90 \times 10^{8}$ \\
\hline 215.605 & 76583 & (e) & $7 / 2$ & 122949 & (o) & $9 / 2$ & 0.02 & $1.49 \times 10^{9}$ \\
\hline 215.818 & 56126 & (e) & $5 / 2$ & 102447 & (o) & $7 / 2$ & 0.32 & $3.03 \times 10^{9}$ \\
\hline 215.910 & 57013 & (e) & $3 / 2$ & 103314 & (o) & $5 / 2$ & 0.15 & $2.02 \times 10^{9}$ \\
\hline 216.318 & 54632 & (e) & $7 / 2$ & 100846 & (o) & $7 / 2$ & 0.31 & $2.87 \times 10^{9}$ \\
\hline 216.732 & 54632 & (e) & $7 / 2$ & 100757 & (o) & $9 / 2$ & 0.39 & $3.47 \times 10^{9}$ \\
\hline 217.809 & 73814 & (e) & $9 / 2$ & 119712 & (o) & $7 / 2$ & -0.49 & $4.60 \times 10^{8}$ \\
\hline 218.452 & 69722 & (e) & $11 / 2$ & 115484 & (o) & $13 / 2$ & 0.14 & $1.95 \times 10^{9}$ \\
\hline 218.599 & 56126 & (e) & $5 / 2$ & 101858 & (o) & $5 / 2$ & -0.11 & $1.07 \times 10^{9}$ \\
\hline 218.761 & 69747 & (e) & $9 / 2$ & 115445 & (o) & $11 / 2$ & 0.25 & $2.50 \times 10^{9}$ \\
\hline 218.769 & 74009 & (e) & $13 / 2$ & 119705 & (o) & $11 / 2$ & -0.32 & $6.61 \times 10^{8}$ \\
\hline 219.120 & 70673 & (e) & $7 / 2$ & 116296 & (o) & $9 / 2$ & -0.48 & $4.66 \times 10^{8}$ \\
\hline 219.603 & 57013 & (e) & $3 / 2$ & 102535 & (o) & $3 / 2$ & -0.41 & $5.40 \times 10^{8}$ \\
\hline 222.498 & 69722 & (e) & $11 / 2$ & 114652 & (o) & $13 / 2$ & -0.01 & $1.31 \times 10^{9}$ \\
\hline 223.533 & 73814 & (e) & $9 / 2$ & 118536 & (o) & $11 / 2$ & -0.27 & $7.22 \times 10^{8}$ \\
\hline 224.072 & 77706 & (e) & $5 / 2$ & 122321 & (o) & $7 / 2$ & -0.44 & $4.81 \times 10^{8}$ \\
\hline 224.608 & 69722 & (e) & $11 / 2$ & 114230 & (o) & $9 / 2$ & -0.16 & $9.16 \times 10^{8}$ \\
\hline 228.681 & 71432 & (e) & $7 / 2$ & 115148 & (o) & $7 / 2$ & -0.43 & $4.76 \times 10^{8}$ \\
\hline 230.332 & 74371 & (e) & $11 / 2$ & 117773 & (o) & $9 / 2$ & -0.45 & $4.49 \times 10^{8}$ \\
\hline 230.354 & 70673 & (e) & $7 / 2$ & 114071 & (o) & $7 / 2$ & -0.35 & $5.59 \times 10^{8}$ \\
\hline
\end{tabular}


Table 4. (Continued.)

\begin{tabular}{|c|c|c|c|c|c|c|c|c|}
\hline \multirow{2}{*}{$\begin{array}{l}\text { Wavelength }{ }^{\mathrm{a}} \\
(\mathrm{nm})\end{array}$} & \multicolumn{3}{|c|}{ Lower level $^{\mathrm{b}}$} & \multicolumn{3}{|c|}{ Upper level $^{\mathrm{b}}$} & \multirow[b]{2}{*}{$\log g f^{\mathrm{c}}$} & \multirow{2}{*}{$\begin{array}{l}g A^{\mathrm{c}} \\
\left(\mathrm{s}^{-1}\right)\end{array}$} \\
\hline & $E\left(\mathrm{~cm}^{-1}\right)$ & Parity & $J$ & $E\left(\mathrm{~cm}^{-1}\right)$ & Parity & $J$ & & \\
\hline 232.313 & 74741 & (e) & $7 / 2$ & 117773 & (o) & $9 / 2$ & -0.35 & $5.58 \times 10^{8}$ \\
\hline 232.792 & 73814 & (e) & $9 / 2$ & 116758 & (o) & $11 / 2$ & 0.05 & $1.38 \times 10^{9}$ \\
\hline 232.838 & 54632 & (e) & $7 / 2$ & 97567 & (o) & $9 / 2$ & -0.22 & $7.45 \times 10^{8}$ \\
\hline 233.853 & 74009 & (e) & $13 / 2$ & 116758 & (o) & $11 / 2$ & -0.31 & $6.06 \times 10^{8}$ \\
\hline 237.484 & 69722 & (e) & $11 / 2$ & 111817 & (o) & $9 / 2$ & -0.44 & $4.23 \times 10^{8}$ \\
\hline 239.656 & 69722 & (e) & $11 / 2$ & 111435 & (o) & $13 / 2$ & -0.11 & $8.97 \times 10^{8}$ \\
\hline 247.067 & 69747 & (e) & $9 / 2$ & 110210 & (o) & $7 / 2$ & -0.36 & $4.71 \times 10^{8}$ \\
\hline
\end{tabular}

a Wavelength (in vacuum (air) below (above) $200 \mathrm{~nm}$ ) deduced from the experimental levels.

${ }^{\mathrm{b}}$ Experimental levels from the NIST compilation (Kramida et al 2012).

c This work (HFR+CPOL calculations).

Table 5. Oscillator strengths and transition probabilities in Pd III $(\log g f>-0.5)$.

\begin{tabular}{|c|c|c|c|c|c|c|c|c|}
\hline \multirow{2}{*}{$\begin{array}{l}\text { Wavelength } \\
(\mathrm{nm})\end{array}$} & \multicolumn{3}{|c|}{ Lower level $^{\mathrm{b}}$} & \multicolumn{3}{|c|}{ Upper level $^{\mathrm{b}}$} & \multirow[b]{2}{*}{$\log g f^{\mathrm{c}}$} & \multirow{2}{*}{$\begin{array}{l}g A^{\mathrm{c}} \\
\left(\mathrm{s}^{-1}\right)\end{array}$} \\
\hline & $E\left(\mathrm{~cm}^{-1}\right)$ & Parity & $J$ & $E\left(\mathrm{~cm}^{-1}\right)$ & Parity & $J$ & & \\
\hline 70.548 & 17880 & (e) & 4 & 159627 & (o) & 3 & -0.06 & $1.16 \times 10^{10}$ \\
\hline 76.305 & 10230 & (e) & 2 & 141283 & (o) & 3 & -0.44 & $4.14 \times 10^{9}$ \\
\hline 77.650 & 17880 & (e) & 4 & 146662 & (o) & 3 & 0.15 & $1.58 \times 10^{10}$ \\
\hline 77.670 & 13469 & (e) & 1 & 142218 & (o) & 2 & -0.44 & $4.03 \times 10^{9}$ \\
\hline 78.102 & 0 & (e) & 4 & 128038 & (o) & 3 & -0.10 & $8.76 \times 10^{9}$ \\
\hline 78.498 & 3229 & (e) & 3 & 130621 & (o) & 2 & -0.48 & $3.61 \times 10^{9}$ \\
\hline 78.730 & 0 & (e) & 4 & 127017 & (o) & 3 & -0.42 & $4.06 \times 10^{9}$ \\
\hline 78.959 & 14634 & (e) & 2 & 141283 & (o) & 3 & -0.35 & $4.77 \times 10^{9}$ \\
\hline 79.407 & 4687 & (e) & 2 & 130621 & (o) & 2 & -0.40 & $4.20 \times 10^{9}$ \\
\hline 79.751 & 0 & (e) & 4 & 125390 & (o) & 5 & 0.08 & $1.26 \times 10^{10}$ \\
\hline 79.901 & 4687 & (e) & 2 & 129841 & (o) & 1 & -0.44 & $3.83 \times 10^{9}$ \\
\hline 79.920 & 10230 & (e) & 2 & 135356 & (o) & 3 & -0.49 & $3.39 \times 10^{9}$ \\
\hline 80.003 & 4687 & (e) & 2 & 129682 & (o) & 3 & -0.13 & $7.71 \times 10^{9}$ \\
\hline 80.011 & 3229 & (e) & 3 & 128212 & (o) & 2 & -0.14 & $7.46 \times 10^{9}$ \\
\hline 80.154 & 0 & (e) & 4 & 124760 & (o) & 5 & -0.01 & $1.01 \times 10^{10}$ \\
\hline 80.157 & 3229 & (e) & 3 & 127984 & (o) & 4 & 0.20 & $1.64 \times 10^{10}$ \\
\hline 80.366 & 0 & (e) & 4 & 124431 & (o) & 4 & -0.24 & $5.99 \times 10^{9}$ \\
\hline 80.368 & 3229 & (e) & 3 & 127658 & (o) & 3 & -0.27 & $5.56 \times 10^{9}$ \\
\hline 81.397 & 14634 & (e) & 2 & 137489 & (o) & 1 & -0.45 & $3.60 \times 10^{9}$ \\
\hline 81.486 & 10230 & (e) & 2 & 132951 & (o) & 2 & -0.46 & $3.50 \times 10^{9}$ \\
\hline 81.505 & 4687 & (e) & 2 & 127378 & (o) & 2 & -0.24 & $5.80 \times 10^{9}$ \\
\hline 81.624 & 10230 & (e) & 2 & 132743 & (o) & 2 & -0.42 & $3.82 \times 10^{9}$ \\
\hline 81.868 & 14634 & (e) & 2 & 136782 & (o) & 1 & -0.30 & $5.04 \times 10^{9}$ \\
\hline 82.535 & 0 & (e) & 4 & 121161 & (o) & 4 & 0.08 & $1.18 \times 10^{10}$ \\
\hline 82.640 & 4687 & (e) & 2 & 125694 & (o) & 3 & -0.27 & $5.19 \times 10^{9}$ \\
\hline 82.640 & 3229 & (e) & 3 & 124236 & (o) & 3 & -0.36 & $4.24 \times 10^{9}$ \\
\hline 82.929 & 14634 & (e) & 2 & 135219 & (o) & 2 & 0.07 & $1.14 \times 10^{10}$ \\
\hline 83.299 & 41698 & (e) & 0 & 161747 & (o) & 1 & -0.38 & $4.00 \times 10^{9}$ \\
\hline 84.057 & 17880 & (e) & 4 & 136847 & (o) & 5 & 0.09 & $1.17 \times 10^{10}$ \\
\hline 84.734 & 41698 & (e) & 0 & 159714 & (o) & 1 & -0.38 & $3.83 \times 10^{9}$ \\
\hline 85.124 & 17880 & (e) & 4 & 135356 & (o) & 3 & -0.46 & $3.18 \times 10^{9}$ \\
\hline 85.172 & 14634 & (e) & 2 & 132044 & (o) & 3 & -0.18 & $6.11 \times 10^{9}$ \\
\hline 85.647 & 17880 & (e) & 4 & 134638 & (o) & 4 & 0.48 & $2.74 \times 10^{10}$ \\
\hline 86.403 & 0 & (e) & 4 & 115736 & (o) & 3 & -0.25 & $5.08 \times 10^{9}$ \\
\hline 88.058 & 3229 & (e) & 3 & 116790 & (o) & 2 & -0.38 & $3.56 \times 10^{9}$ \\
\hline 88.590 & 4687 & (e) & 2 & 117566 & (o) & 1 & -0.69 & $1.75 \times 10^{9}$ \\
\hline 88.929 & 0 & (e) & 4 & 112449 & (o) & 4 & -0.18 & $5.57 \times 10^{9}$ \\
\hline 90.048 & 4687 & (e) & 2 & 115738 & (o) & 2 & -0.45 & $2.95 \times 10^{9}$ \\
\hline 90.439 & 3229 & (e) & 3 & 113802 & (o) & 3 & -0.40 & $3.27 \times 10^{9}$ \\
\hline 167.128 & 78581 & (e) & 4 & 138415 & (o) & 3 & -0.49 & $7.78 \times 10^{8}$ \\
\hline 168.818 & 75403 & (e) & 4 & 134638 & (o) & 4 & -0.48 & $7.73 \times 10^{8}$ \\
\hline 168.926 & 103548 & (e) & 2 & 162746 & (o) & 2 & -0.49 & $7.47 \times 10^{8}$ \\
\hline 169.343 & 104418 & (e) & 3 & 163470 & (o) & 3 & 0.29 & $4.51 \times 10^{9}$ \\
\hline 170.380 & 103109 & (e) & 1 & 161801 & (o) & 2 & -0.37 & $9.68 \times 10^{8}$ \\
\hline 170.538 & 103109 & (e) & 1 & 161747 & (o) & 1 & -0.38 & $9.65 \times 10^{8}$ \\
\hline 170.857 & 65708 & (e) & 3 & 124236 & (o) & 3 & -0.45 & $8.12 \times 10^{8}$ \\
\hline 171.665 & 103548 & (e) & 2 & 161801 & (o) & 2 & -0.04 & $2.06 \times 10^{9}$ \\
\hline
\end{tabular}


Table 5. (Continued.)

\begin{tabular}{|c|c|c|c|c|c|c|c|c|}
\hline \multirow{2}{*}{$\begin{array}{l}\text { Wavelength }{ }^{\mathrm{a}} \\
(\mathrm{nm})\end{array}$} & \multicolumn{3}{|c|}{ Lower level $^{\mathrm{b}}$} & \multicolumn{3}{|c|}{ Upper level $^{\mathrm{b}}$} & \multirow[b]{2}{*}{$\log g f^{\mathrm{c}}$} & \multirow{2}{*}{$\begin{array}{l}g A^{\mathrm{c}} \\
\left(\mathrm{s}^{-1}\right)\end{array}$} \\
\hline & $E\left(\mathrm{~cm}^{-1}\right)$ & Parity & $J$ & $E\left(\mathrm{~cm}^{-1}\right)$ & Parity & $J$ & & \\
\hline 171.985 & 56742 & (e) & 3 & 114886 & (o) & 4 & -0.17 & $1.54 \times 10^{9}$ \\
\hline 172.273 & 75403 & (e) & 4 & 133450 & (o) & 4 & 0.05 & $2.50 \times 10^{9}$ \\
\hline 172.440 & 65788 & (e) & 2 & 123779 & (o) & 1 & -0.49 & $7.20 \times 10^{8}$ \\
\hline 173.024 & 65708 & (e) & 3 & 123503 & (o) & 3 & -0.49 & $7.25 \times 10^{8}$ \\
\hline 173.264 & 65788 & (e) & 2 & 123503 & (o) & 3 & 0.21 & $3.60 \times 10^{9}$ \\
\hline 174.161 & 55089 & (e) & 4 & 112507 & (o) & 5 & 0.25 & $3.96 \times 10^{9}$ \\
\hline 174.555 & 67151 & (e) & 1 & 124439 & (o) & 2 & -0.16 & $1.53 \times 10^{9}$ \\
\hline 174.809 & 65788 & (e) & 2 & 122993 & (o) & 2 & -0.49 & $7.10 \times 10^{8}$ \\
\hline 175.632 & 71047 & (e) & 4 & 127984 & (o) & 4 & -0.38 & $9.06 \times 10^{8}$ \\
\hline 175.819 & 74674 & (e) & 6 & 131550 & (o) & 6 & 0.60 & $8.57 \times 10^{9}$ \\
\hline 176.422 & 85420 & (e) & 2 & 142103 & (o) & 1 & -0.06 & $1.88 \times 10^{9}$ \\
\hline 176.540 & 65255 & (e) & 3 & 121899 & (o) & 4 & -0.09 & $1.76 \times 10^{9}$ \\
\hline 176.664 & 103109 & (e) & 1 & 159714 & (o) & 1 & -0.30 & $1.07 \times 10^{9}$ \\
\hline 177.341 & 85830 & (e) & 3 & 142218 & (o) & 2 & 0.02 & $2.18 \times 10^{9}$ \\
\hline 177.516 & 75967 & (e) & 5 & 132300 & (o) & 5 & 0.53 & $7.20 \times 10^{9}$ \\
\hline 177.963 & 65708 & (e) & 3 & 121899 & (o) & 4 & 0.28 & $4.00 \times 10^{9}$ \\
\hline 178.062 & 52916 & (e) & 5 & 109076 & (o) & 5 & -0.38 & $8.74 \times 10^{8}$ \\
\hline 178.096 & 85830 & (e) & 3 & 141979 & (o) & 4 & -0.21 & $1.31 \times 10^{9}$ \\
\hline 178.126 & 71047 & (e) & 4 & 127187 & (o) & 5 & -0.08 & $1.74 \times 10^{9}$ \\
\hline 178.255 & 52916 & (e) & 5 & 109016 & (o) & 6 & 0.70 & $1.05 \times 10^{10}$ \\
\hline 178.322 & 103548 & (e) & 2 & 159627 & (o) & 3 & -0.27 & $1.12 \times 10^{9}$ \\
\hline 178.388 & 78581 & (e) & 4 & 134638 & (o) & 4 & 0.08 & $2.53 \times 10^{9}$ \\
\hline 178.437 & 80804 & (e) & 5 & 136847 & (o) & 5 & 0.56 & $7.50 \times 10^{9}$ \\
\hline 178.640 & 90684 & (e) & 3 & 146662 & (o) & 3 & 0.36 & $4.78 \times 10^{9}$ \\
\hline 178.998 & 71047 & (e) & 4 & 126914 & (o) & 4 & -0.14 & $1.50 \times 10^{9}$ \\
\hline 179.509 & 56742 & (e) & 3 & 112449 & (o) & 4 & -0.37 & $8.77 \times 10^{8}$ \\
\hline 179.661 & 55089 & (e) & 4 & 110749 & (o) & 4 & -0.80 & $3.25 \times 10^{8}$ \\
\hline 179.912 & 75967 & (e) & 5 & 131550 & (o) & 6 & -0.39 & $8.43 \times 10^{8}$ \\
\hline 180.334 & 85830 & (e) & 3 & 141283 & (o) & 3 & -0.43 & $7.62 \times 10^{8}$ \\
\hline 180.418 & 72786 & (e) & 3 & 128212 & (o) & 2 & -0.35 & $9.10 \times 10^{8}$ \\
\hline 180.491 & 69985 & (e) & 5 & 125390 & (o) & 5 & 0.39 & $5.03 \times 10^{9}$ \\
\hline 180.854 & 72745 & (e) & 2 & 128038 & (o) & 3 & 0.18 & $3.09 \times 10^{9}$ \\
\hline 181.197 & 85830 & (e) & 3 & 141019 & (o) & 4 & 0.21 & $3.28 \times 10^{9}$ \\
\hline 181.210 & 86795 & (e) & 4 & 141979 & (o) & 4 & 0.20 & $3.22 \times 10^{9}$ \\
\hline 181.352 & 85420 & (e) & 2 & 140562 & (o) & 2 & 0.02 & $2.11 \times 10^{9}$ \\
\hline 181.557 & 86795 & (e) & 4 & 141874 & (o) & 5 & 0.56 & $7.26 \times 10^{9}$ \\
\hline 181.645 & 85420 & (e) & 2 & 140473 & (o) & 3 & -0.15 & $1.42 \times 10^{9}$ \\
\hline 181.847 & 75967 & (e) & 5 & 130959 & (o) & 6 & 0.07 & $2.38 \times 10^{9}$ \\
\hline 181.928 & 65255 & (e) & 3 & 120222 & (o) & 3 & -0.32 & $9.53 \times 10^{8}$ \\
\hline 182.251 & 78581 & (e) & 4 & 133450 & (o) & 4 & 0.17 & $2.99 \times 10^{9}$ \\
\hline 182.251 & 82620 & (e) & 1 & 137489 & (o) & 1 & -0.24 & $1.15 \times 10^{9}$ \\
\hline 183.006 & 85830 & (e) & 3 & 140473 & (o) & 3 & -0.07 & $1.68 \times 10^{9}$ \\
\hline 183.176 & 72786 & (e) & 3 & 127378 & (o) & 2 & 0.04 & $2.16 \times 10^{9}$ \\
\hline 183.208 & 67151 & (e) & 1 & 121734 & (o) & 1 & -0.28 & $1.03 \times 10^{9}$ \\
\hline 183.330 & 78169 & (e) & 2 & 132716 & (o) & 1 & -0.26 & $1.10 \times 10^{9}$ \\
\hline 183.439 & 65708 & (e) & 3 & 120222 & (o) & 3 & -0.27 & $1.05 \times 10^{9}$ \\
\hline 183.527 & 86795 & (e) & 4 & 141283 & (o) & 3 & 0.12 & $2.58 \times 10^{9}$ \\
\hline 183.668 & 69985 & (e) & 5 & 124431 & (o) & 4 & -0.05 & $1.74 \times 10^{9}$ \\
\hline 183.708 & 65788 & (e) & 2 & 120222 & (o) & 3 & -0.33 & $9.28 \times 10^{8}$ \\
\hline 183.861 & 108357 & (e) & 2 & 162746 & (o) & 2 & 0.12 & $2.62 \times 10^{9}$ \\
\hline 184.017 & 71047 & (e) & 4 & 125390 & (o) & 5 & -0.32 & $9.47 \times 10^{8}$ \\
\hline 184.231 & 75403 & (e) & 4 & 129682 & (o) & 3 & -0.39 & $8.00 \times 10^{8}$ \\
\hline 184.315 & 65788 & (e) & 2 & 120043 & (o) & 2 & -0.29 & $1.01 \times 10^{9}$ \\
\hline 184.349 & 76231 & (e) & 3 & 130476 & (o) & 4 & 0.49 & $6.06 \times 10^{9}$ \\
\hline 184.395 & 72786 & (e) & 3 & 127017 & (o) & 3 & 0.07 & $2.32 \times 10^{9}$ \\
\hline 184.748 & 72786 & (e) & 3 & 126914 & (o) & 4 & 0.03 & $2.07 \times 10^{9}$ \\
\hline 185.160 & 56742 & (e) & 3 & 110749 & (o) & 4 & 0.33 & $4.18 \times 10^{9}$ \\
\hline 185.227 & 55089 & (e) & 4 & 109076 & (o) & 5 & 0.35 & $4.40 \times 10^{9}$ \\
\hline 185.617 & 78169 & (e) & 2 & 132044 & (o) & 3 & 0.06 & $2.20 \times 10^{9}$ \\
\hline 185.757 & 80804 & (e) & 5 & 134638 & (o) & 4 & 0.17 & $2.90 \times 10^{9}$ \\
\hline 185.922 & 57845 & (e) & 2 & 111631 & (o) & 3 & 0.30 & $3.88 \times 10^{9}$ \\
\hline 185.985 & 104418 & (e) & 3 & 158186 & (o) & 4 & 0.49 & $5.92 \times 10^{9}$ \\
\hline 186.296 & 86795 & (e) & 4 & 140473 & (o) & 3 & -0.44 & $6.91 \times 10^{8}$ \\
\hline 186.579 & 71047 & (e) & 4 & 124644 & (o) & 3 & -0.22 & $1.15 \times 10^{9}$ \\
\hline
\end{tabular}


Table 5. (Continued.)

\begin{tabular}{|c|c|c|c|c|c|c|c|c|}
\hline \multirow{2}{*}{$\begin{array}{l}\text { Wavelength } \\
(\mathrm{nm})\end{array}$} & \multicolumn{3}{|c|}{ Lower level $^{\text {b }}$} & \multicolumn{3}{|c|}{ Upper level $^{\mathrm{b}}$} & \multirow[b]{2}{*}{$\log g f^{\mathrm{c}}$} & \multirow{2}{*}{$\begin{array}{l}g A^{\mathrm{c}} \\
\left(\mathrm{s}^{-1}\right)\end{array}$} \\
\hline & $E\left(\mathrm{~cm}^{-1}\right)$ & Parity & $J$ & $E\left(\mathrm{~cm}^{-1}\right)$ & Parity & $J$ & & \\
\hline 186.643 & 83204 & (e) & 2 & 136782 & (o) & 1 & -0.07 & $1.61 \times 10^{9}$ \\
\hline 187.044 & 78581 & (e) & 4 & 132044 & (o) & 3 & -0.11 & $1.47 \times 10^{9}$ \\
\hline 187.126 & 65788 & (e) & 2 & 119228 & (o) & 1 & -0.48 & $6.33 \times 10^{8}$ \\
\hline 187.301 & 108357 & (e) & 2 & 161747 & (o) & 1 & -0.27 & $1.03 \times 10^{9}$ \\
\hline 187.321 & 71047 & (e) & 4 & 124431 & (o) & 4 & 0.00 & $1.91 \times 10^{9}$ \\
\hline 187.461 & 69985 & (e) & 5 & 123330 & (o) & 6 & 0.66 & $8.65 \times 10^{9}$ \\
\hline 187.705 & 58527 & (e) & 1 & 111802 & (o) & 2 & 0.17 & $2.84 \times 10^{9}$ \\
\hline 187.905 & 85830 & (e) & 3 & 139048 & (o) & 4 & -0.26 & $1.05 \times 10^{9}$ \\
\hline 188.008 & 71047 & (e) & 4 & 124236 & (o) & 3 & -0.01 & $1.85 \times 10^{9}$ \\
\hline 188.033 & 58527 & (e) & 1 & 111710 & (o) & 1 & -0.21 & $1.17 \times 10^{9}$ \\
\hline 188.337 & 57845 & (e) & 2 & 110941 & (o) & 2 & 0.11 & $2.40 \times 10^{9}$ \\
\hline 188.583 & 55089 & (e) & 4 & 108116 & (o) & 4 & 0.42 & $4.96 \times 10^{9}$ \\
\hline 188.698 & 85420 & (e) & 2 & 138415 & (o) & 3 & -0.03 & $1.76 \times 10^{9}$ \\
\hline 188.741 & 56742 & (e) & 3 & 109724 & (o) & 3 & 0.29 & $3.65 \times 10^{9}$ \\
\hline 189.134 & 74674 & (e) & 6 & 127546 & (o) & 7 & 0.75 & $1.04 \times 10^{10}$ \\
\hline 190.168 & 85830 & (e) & 3 & 138415 & (o) & 3 & -0.09 & $1.51 \times 10^{9}$ \\
\hline 190.174 & 75455 & (e) & 2 & 128038 & (o) & 3 & -0.45 & $6.56 \times 10^{8}$ \\
\hline 190.472 & 78120 & (e) & 1 & 130621 & (o) & 2 & -0.26 & $1.02 \times 10^{9}$ \\
\hline 190.613 & 103548 & (e) & 2 & 156011 & (o) & 3 & 0.20 & $2.91 \times 10^{9}$ \\
\hline 191.281 & 103109 & (e) & 1 & 155388 & (o) & 0 & -0.46 & $6.33 \times 10^{8}$ \\
\hline 191.375 & 86795 & (e) & 4 & 139048 & (o) & 4 & 0.09 & $2.23 \times 10^{9}$ \\
\hline 191.462 & 52916 & (e) & 5 & 105146 & (o) & 5 & 0.54 & $6.27 \times 10^{9}$ \\
\hline 191.729 & 76055 & (e) & 1 & 128212 & (o) & 2 & -0.10 & $1.46 \times 10^{9}$ \\
\hline 191.748 & 83204 & (e) & 2 & 135356 & (o) & 3 & 0.18 & $2.73 \times 10^{9}$ \\
\hline 192.245 & 75967 & (e) & 5 & 127984 & (o) & 4 & 0.22 & $2.99 \times 10^{9}$ \\
\hline 192.252 & 83204 & (e) & 2 & 135219 & (o) & 2 & -0.13 & $1.34 \times 10^{9}$ \\
\hline 192.535 & 75967 & (e) & 5 & 127906 & (o) & 5 & -0.48 & $6.05 \times 10^{8}$ \\
\hline 192.548 & 57845 & (e) & 2 & 109780 & (o) & 1 & -0.17 & $1.22 \times 10^{9}$ \\
\hline 192.678 & 56742 & (e) & 3 & 108642 & (o) & 2 & 0.12 & $2.36 \times 10^{9}$ \\
\hline 193.033 & 55089 & (e) & 4 & 106893 & (o) & 3 & 0.29 & $3.50 \times 10^{9}$ \\
\hline 193.108 & 75403 & (e) & 4 & 127187 & (o) & 5 & 0.45 & $4.99 \times 10^{9}$ \\
\hline 193.272 & 85420 & (e) & 2 & 137161 & (o) & 2 & -0.36 & $7.74 \times 10^{8}$ \\
\hline 193.342 & 78120 & (e) & 1 & 129841 & (o) & 1 & -0.47 & $5.96 \times 10^{8}$ \\
\hline 193.827 & 104418 & (e) & 3 & 156011 & (o) & 3 & -0.41 & $6.94 \times 10^{8}$ \\
\hline 194.045 & 90684 & (e) & 3 & 142218 & (o) & 2 & -0.39 & $7.28 \times 10^{8}$ \\
\hline 194.127 & 78169 & (e) & 2 & 129682 & (o) & 3 & -0.23 & $1.05 \times 10^{9}$ \\
\hline 194.133 & 75403 & (e) & 4 & 126914 & (o) & 4 & -0.41 & $6.94 \times 10^{8}$ \\
\hline 194.164 & 52916 & (e) & 5 & 104419 & (o) & 4 & 0.40 & $4.43 \times 10^{9}$ \\
\hline 194.453 & 76231 & (e) & 3 & 127658 & (o) & 3 & 0.04 & $1.91 \times 10^{9}$ \\
\hline 194.716 & 108357 & (e) & 2 & 159714 & (o) & 1 & -0.34 & $8.17 \times 10^{8}$ \\
\hline 194.820 & 103109 & (e) & 1 & 154438 & (o) & 2 & 0.09 & $2.15 \times 10^{9}$ \\
\hline 194.904 & 78534 & (e) & 0 & 129841 & (o) & 1 & -0.27 & $9.42 \times 10^{8}$ \\
\hline 194.949 & 90684 & (e) & 3 & 141979 & (o) & 4 & -0.11 & $1.36 \times 10^{9}$ \\
\hline 195.046 & 108357 & (e) & 2 & 159627 & (o) & 3 & 0.27 & $3.26 \times 10^{9}$ \\
\hline 195.156 & 62561 & (e) & 4 & 113802 & (o) & 3 & 0.21 & $2.83 \times 10^{9}$ \\
\hline 195.404 & 69985 & (e) & 5 & 121161 & (o) & 4 & 0.22 & $2.89 \times 10^{9}$ \\
\hline 195.688 & 78581 & (e) & 4 & 129682 & (o) & 3 & -0.10 & $1.38 \times 10^{9}$ \\
\hline 195.719 & 76055 & (e) & 1 & 127149 & (o) & 1 & -0.45 & $6.25 \times 10^{8}$ \\
\hline 196.007 & 103548 & (e) & 2 & 154567 & (o) & 1 & -0.04 & $1.57 \times 10^{9}$ \\
\hline 196.286 & 75967 & (e) & 5 & 126914 & (o) & 4 & -0.15 & $1.23 \times 10^{9}$ \\
\hline 196.905 & 76231 & (e) & 3 & 127017 & (o) & 3 & -0.35 & $7.71 \times 10^{8}$ \\
\hline 197.175 & 74674 & (e) & 6 & 125390 & (o) & 5 & -0.22 & $1.04 \times 10^{9}$ \\
\hline 197.230 & 69985 & (e) & 5 & 120688 & (o) & 5 & 0.01 & $1.76 \times 10^{9}$ \\
\hline 197.258 & 74281 & (e) & 0 & 124976 & (o) & 1 & -0.47 & $5.77 \times 10^{8}$ \\
\hline 197.519 & 78169 & (e) & 2 & 128798 & (o) & 2 & -0.08 & $1.43 \times 10^{9}$ \\
\hline 197.535 & 75455 & (e) & 2 & 126079 & (o) & 2 & -0.24 & $9.89 \times 10^{8}$ \\
\hline 197.753 & 72745 & (e) & 2 & 123313 & (o) & 2 & 0.03 & $1.84 \times 10^{9}$ \\
\hline 198.061 & 74674 & (e) & 6 & 125163 & (o) & 6 & -0.36 & $7.50 \times 10^{8}$ \\
\hline 198.069 & 67079 & (e) & 2 & 117566 & (o) & 1 & -0.24 & $9.90 \times 10^{8}$ \\
\hline 198.087 & 65255 & (e) & 3 & 115738 & (o) & 2 & -0.20 & $1.07 \times 10^{9}$ \\
\hline 198.094 & 65255 & (e) & 3 & 115736 & (o) & 3 & -0.02 & $1.61 \times 10^{9}$ \\
\hline 198.489 & 78120 & (e) & 1 & 128500 & (o) & 1 & -0.47 & $5.72 \times 10^{8}$ \\
\hline 198.671 & 90684 & (e) & 3 & 141019 & (o) & 4 & 0.14 & $2.37 \times 10^{9}$ \\
\hline 198.686 & 82620 & (e) & 1 & 132951 & (o) & 2 & -0.09 & $1.37 \times 10^{9}$ \\
\hline
\end{tabular}


Table 5. (Continued.)

\begin{tabular}{|c|c|c|c|c|c|c|c|c|}
\hline \multirow{2}{*}{$\begin{array}{l}\text { Wavelength }{ }^{\mathrm{a}} \\
(\mathrm{nm})\end{array}$} & \multicolumn{3}{|c|}{ Lower level $^{\text {b }}$} & \multicolumn{3}{|c|}{ Upper level $^{\mathrm{b}}$} & \multirow[b]{2}{*}{$\log g f^{\mathrm{c}}$} & \multirow{2}{*}{$\begin{array}{l}g A^{\mathrm{c}} \\
\left(\mathrm{s}^{-1}\right)\end{array}$} \\
\hline & $E\left(\mathrm{~cm}^{-1}\right)$ & Parity & $J$ & $E\left(\mathrm{~cm}^{-1}\right)$ & Parity & $J$ & & \\
\hline 198.841 & 75403 & (e) & 4 & 125694 & (o) & 3 & 0.01 & $1.71 \times 10^{9}$ \\
\hline 199.384 & 80804 & (e) & 5 & 130959 & (o) & 6 & 0.54 & $5.78 \times 10^{9}$ \\
\hline 199.511 & 82620 & (e) & 1 & 132743 & (o) & 2 & -0.42 & $6.41 \times 10^{8}$ \\
\hline 199.544 & 71047 & (e) & 4 & 121161 & (o) & 4 & -0.34 & $7.70 \times 10^{8}$ \\
\hline 199.655 & 74674 & (e) & 6 & 124760 & (o) & 5 & 0.43 & $4.49 \times 10^{9}$ \\
\hline 199.880 & 65708 & (e) & 3 & 115738 & (o) & 2 & -0.42 & $6.31 \times 10^{8}$ \\
\hline 199.887 & 65708 & (e) & 3 & 115736 & (o) & 3 & -0.30 & $8.45 \times 10^{8}$ \\
\hline 199.910 & 75455 & (e) & 2 & 125477 & (o) & 1 & -0.33 & $7.87 \times 10^{8}$ \\
\hline 199.975 & 73003 & (e) & 1 & 122993 & (o) & 2 & -0.25 & $9.42 \times 10^{8}$ \\
\hline 199.991 & 72786 & (e) & 3 & 122772 & (o) & 4 & 0.27 & $3.08 \times 10^{9}$ \\
\hline 200.151 & 62561 & (e) & 4 & 112507 & (o) & 5 & 0.32 & $3.46 \times 10^{9}$ \\
\hline 200.383 & 62561 & (e) & 4 & 112449 & (o) & 4 & 0.35 & $3.71 \times 10^{9}$ \\
\hline 200.425 & 90684 & (e) & 3 & 140562 & (o) & 2 & -0.32 & $8.06 \times 10^{8}$ \\
\hline 200.547 & 76231 & (e) & 3 & 126079 & (o) & 2 & -0.42 & $6.27 \times 10^{8}$ \\
\hline 201.096 & 67079 & (e) & 2 & 116790 & (o) & 2 & -0.15 & $1.17 \times 10^{9}$ \\
\hline 201.383 & 71047 & (e) & 4 & 120688 & (o) & 5 & 0.37 & $3.85 \times 10^{9}$ \\
\hline 201.422 & 65255 & (e) & 3 & 114886 & (o) & 4 & 0.23 & $2.77 \times 10^{9}$ \\
\hline 201.797 & 83204 & (e) & 2 & 132743 & (o) & 2 & -0.21 & $1.01 \times 10^{9}$ \\
\hline 201.915 & 104418 & (e) & 3 & 153928 & (o) & 2 & 0.19 & $2.52 \times 10^{9}$ \\
\hline 202.200 & 67079 & (e) & 2 & 116519 & (o) & 3 & 0.23 & $2.78 \times 10^{9}$ \\
\hline 202.539 & 75403 & (e) & 4 & 124760 & (o) & 5 & -0.44 & $5.90 \times 10^{8}$ \\
\hline 202.670 & 78581 & (e) & 4 & 127906 & (o) & 5 & 0.49 & $5.07 \times 10^{9}$ \\
\hline 203.205 & 75967 & (e) & 5 & 125163 & (o) & 6 & 0.48 & $4.87 \times 10^{9}$ \\
\hline 203.232 & 75455 & (e) & 2 & 124644 & (o) & 3 & -0.11 & $1.26 \times 10^{9}$ \\
\hline 203.277 & 65708 & (e) & 3 & 114886 & (o) & 4 & -0.22 & $9.78 \times 10^{8}$ \\
\hline 203.896 & 75403 & (e) & 4 & 124431 & (o) & 4 & -0.43 & $5.89 \times 10^{8}$ \\
\hline 204.079 & 75455 & (e) & 2 & 124439 & (o) & 2 & -0.43 & $5.96 \times 10^{8}$ \\
\hline 205.445 & 67079 & (e) & 2 & 115738 & (o) & 2 & -0.42 & $6.03 \times 10^{8}$ \\
\hline 206.698 & 90684 & (e) & 3 & 139048 & (o) & 4 & -0.08 & $1.30 \times 10^{9}$ \\
\hline 211.041 & 75403 & (e) & 4 & 122772 & (o) & 4 & -0.31 & $7.28 \times 10^{8}$ \\
\hline 211.888 & 80804 & (e) & 5 & 127984 & (o) & 4 & -0.30 & $7.42 \times 10^{8}$ \\
\hline 212.368 & 65255 & (e) & 3 & 112328 & (o) & 2 & -0.42 & $5.67 \times 10^{8}$ \\
\hline 214.431 & 65708 & (e) & 3 & 112328 & (o) & 2 & -0.18 & $9.71 \times 10^{8}$ \\
\hline 214.799 & 65788 & (e) & 2 & 112328 & (o) & 2 & -0.35 & $6.47 \times 10^{8}$ \\
\hline 214.914 & 62561 & (e) & 4 & 109076 & (o) & 5 & 0.07 & $1.71 \times 10^{9}$ \\
\hline 215.093 & 90684 & (e) & 3 & 137161 & (o) & 2 & -0.20 & $9.05 \times 10^{8}$ \\
\hline 219.742 & 65255 & (e) & 3 & 110749 & (o) & 4 & -0.37 & $5.88 \times 10^{8}$ \\
\hline 225.365 & 80804 & (e) & 5 & 125163 & (o) & 6 & -0.07 & $1.10 \times 10^{9}$ \\
\hline 229.145 & 80804 & (e) & 5 & 124431 & (o) & 4 & -0.41 & $4.93 \times 10^{8}$ \\
\hline 234.753 & 62561 & (e) & 4 & 105146 & (o) & 5 & -0.49 & $3.87 \times 10^{8}$ \\
\hline
\end{tabular}

a Wavelength (in vacuum (air) below (above) $200 \mathrm{~nm}$ ) deduced from the experimental levels.

${ }^{\mathrm{b}}$ Experimental levels from the NIST compilation (Kramida et al 2012).

${ }^{c}$ This work (HFR+CPOL calculations).

Table 6. Oscillator strengths and transition probabilities in Ag III $(\log g f>-0.5)$.

\begin{tabular}{|c|c|c|c|c|c|c|c|c|}
\hline \multirow{2}{*}{$\begin{array}{l}\text { Wavelength } \\
\text { (nm) }\end{array}$} & \multicolumn{3}{|c|}{ Lower level $^{\mathrm{b}}$} & \multicolumn{3}{|c|}{ Upper level $^{\mathrm{b}}$} & \multirow[b]{2}{*}{$\log g f^{\mathrm{c}}$} & \multirow{2}{*}{$\begin{array}{l}g A^{\mathrm{c}} \\
\left(\mathrm{s}^{-1}\right)\end{array}$} \\
\hline & $E\left(\mathrm{~cm}^{-1}\right)$ & Parity & $J$ & $E\left(\mathrm{~cm}^{-1}\right)$ & Parity & $J$ & & \\
\hline 71.390 & 0 & (e) & $5 / 2$ & 140076 & (o) & $3 / 2$ & -0.25 & $7.34 \times 10^{9}$ \\
\hline 71.854 & 4609 & (e) & $3 / 2$ & 143781 & (o) & $1 / 2$ & -0.22 & $7.80 \times 10^{9}$ \\
\hline 72.699 & 4609 & (e) & $3 / 2$ & 142164 & (o) & $5 / 2$ & -0.03 & $1.19 \times 10^{10}$ \\
\hline 73.007 & 0 & (e) & $5 / 2$ & 136974 & (o) & $7 / 2$ & 0.02 & $1.31 \times 10^{10}$ \\
\hline 73.660 & 0 & (e) & $5 / 2$ & 135759 & (o) & $5 / 2$ & -0.41 & $4.80 \times 10^{9}$ \\
\hline 74.101 & 0 & (e) & $5 / 2$ & 134952 & (o) & $3 / 2$ & -0.43 & $4.49 \times 10^{9}$ \\
\hline 74.233 & 4609 & (e) & $3 / 2$ & 139321 & (o) & $3 / 2$ & -0.16 & $8.44 \times 10^{9}$ \\
\hline 76.721 & 4609 & (e) & $3 / 2$ & 134952 & (o & $3 / 2$ & -0.44 & $4.11 \times 10^{9}$ \\
\hline 76.836 & 0 & (e) & $5 / 2$ & 48 & (o & $5 / 2$ & -0.37 & $4.78 \times 10^{9}$ \\
\hline 77.640 & 0 & (e) & $5 / 2$ & 128799 & (o) & $5 / 2$ & -0.22 & $6.67 \times 10^{9}$ \\
\hline 79.794 & 4609 & (e) & $3 / 2$ & 129933 & (o) & $3 / 2$ & -0.45 & $3.74 \times 10^{9}$ \\
\hline 79.943 & 0 & (e) & $5 / 2$ & 125090 & (o) & $5 / 2$ & -0.34 & $4.78 \times 10^{9}$ \\
\hline 158.740 & 78893 & (e) & $1 / 2$ & 141890 & (o) & $3 / 2$ & -0.45 & $9.31 \times 10^{8}$ \\
\hline
\end{tabular}


Table 6. (Continued.)

\begin{tabular}{|c|c|c|c|c|c|c|c|c|}
\hline \multirow{2}{*}{$\begin{array}{l}\text { Wavelength }{ }^{\mathrm{a}} \\
(\mathrm{nm})\end{array}$} & \multicolumn{3}{|c|}{ Lower level $^{\mathrm{b}}$} & \multicolumn{3}{|c|}{ Upper level $^{\mathrm{b}}$} & \multirow[b]{2}{*}{$\log g f^{\mathrm{c}}$} & \multirow{2}{*}{$\begin{array}{l}g A^{\mathrm{c}} \\
\left(\mathrm{s}^{-1}\right)\end{array}$} \\
\hline & $E\left(\mathrm{~cm}^{-1}\right)$ & Parity & $J$ & $E\left(\mathrm{~cm}^{-1}\right)$ & Parity & $J$ & & \\
\hline 165.094 & 76402 & (e) & $5 / 2$ & 136974 & (o) & $7 / 2$ & -0.31 & $1.19 \times 10^{9}$ \\
\hline 165.711 & 85724 & (e) & $7 / 2$ & 146070 & (o) & $9 / 2$ & -0.36 & $1.07 \times 10^{9}$ \\
\hline 167.076 & 85724 & (e) & $7 / 2$ & 145577 & (o) & $7 / 2$ & 0.45 & $6.73 \times 10^{9}$ \\
\hline 167.496 & 73929 & (e) & $5 / 2$ & 133632 & (o) & $7 / 2$ & -0.22 & $1.44 \times 10^{9}$ \\
\hline 167.825 & 68139 & (e) & $5 / 2$ & 127725 & (o) & $7 / 2$ & -0.13 & $1.78 \times 10^{9}$ \\
\hline 168.105 & 65759 & (e) & $7 / 2$ & 125246 & (o) & $9 / 2$ & 0.13 & $3.17 \times 10^{9}$ \\
\hline 169.354 & 63246 & (e) & $9 / 2$ & 122294 & (o) & $9 / 2$ & 0.35 & $5.20 \times 10^{9}$ \\
\hline 170.303 & 80127 & (e) & $3 / 2$ & 138846 & (o) & $5 / 2$ & -0.47 & $7.72 \times 10^{8}$ \\
\hline 170.505 & 82228 & (e) & $5 / 2$ & 140878 & (o) & $7 / 2$ & 0.25 & $4.01 \times 10^{9}$ \\
\hline 170.646 & 85180 & (e) & $3 / 2$ & 143781 & (o) & $1 / 2$ & -0.23 & $1.35 \times 10^{9}$ \\
\hline 170.884 & 69346 & (e) & $3 / 2$ & 127865 & (o) & $3 / 2$ & -0.29 & $1.16 \times 10^{9}$ \\
\hline 172.226 & 68139 & (e) & $5 / 2$ & 126203 & (o) & $5 / 2$ & -0.07 & $1.94 \times 10^{9}$ \\
\hline 172.589 & 77409 & (e) & $3 / 2$ & 135350 & (o) & $3 / 2$ & -0.26 & $1.25 \times 10^{9}$ \\
\hline 172.812 & 65759 & (e) & $7 / 2$ & 123626 & (o) & $7 / 2$ & -0.06 & $1.94 \times 10^{9}$ \\
\hline 174.735 & 76402 & (e) & $5 / 2$ & 133632 & (o) & $7 / 2$ & 0.17 & $3.20 \times 10^{9}$ \\
\hline 174.964 & 111436 & (e) & $1 / 2$ & 168591 & (o) & $3 / 2$ & 0.14 & $3.03 \times 10^{9}$ \\
\hline 175.114 & 63246 & (e) & $9 / 2$ & 120352 & (o) & $11 / 2$ & 0.65 & $9.80 \times 10^{9}$ \\
\hline 176.059 & 80127 & (e) & $3 / 2$ & 136926 & (o) & $5 / 2$ & 0.02 & $2.24 \times 10^{9}$ \\
\hline 176.263 & 78893 & (e) & $1 / 2$ & 135627 & (o) & $1 / 2$ & -0.33 & $1.02 \times 10^{9}$ \\
\hline 176.436 & 80127 & (e) & $3 / 2$ & 136805 & (o) & $3 / 2$ & -0.29 & $1.09 \times 10^{9}$ \\
\hline 176.881 & 65759 & (e) & $7 / 2$ & 122294 & (o) & $9 / 2$ & -0.09 & $1.72 \times 10^{9}$ \\
\hline 177.127 & 78893 & (e) & $1 / 2$ & 135350 & (o) & $3 / 2$ & -0.39 & $8.76 \times 10^{8}$ \\
\hline 177.181 & 85724 & (e) & $7 / 2$ & 142164 & (o) & $5 / 2$ & 0.21 & $3.50 \times 10^{9}$ \\
\hline 179.391 & 69346 & (e) & $3 / 2$ & 125090 & (o) & $5 / 2$ & -0.05 & $1.88 \times 10^{9}$ \\
\hline 180.224 & 68139 & (e) & $5 / 2$ & 123626 & (o) & $7 / 2$ & -0.05 & $1.85 \times 10^{9}$ \\
\hline 180.226 & 85506 & (e) & $1 / 2$ & 140991 & (o) & $1 / 2$ & -0.34 & $9.43 \times 10^{8}$ \\
\hline 180.823 & 65759 & (e) & $7 / 2$ & 121062 & (o) & $7 / 2$ & 0.21 & $3.28 \times 10^{9}$ \\
\hline 180.892 & 85596 & (e) & $9 / 2$ & 140878 & (o) & $7 / 2$ & -0.04 & $1.87 \times 10^{9}$ \\
\hline 181.684 & 71686 & (e) & $7 / 2$ & 126727 & (o) & $7 / 2$ & 0.19 & $3.11 \times 10^{9}$ \\
\hline 182.247 & 73929 & (e) & $5 / 2$ & 128799 & (o) & $5 / 2$ & 0.05 & $2.22 \times 10^{9}$ \\
\hline 182.401 & 80127 & (e) & $3 / 2$ & 134952 & (o) & $3 / 2$ & -0.45 & $7.00 \times 10^{8}$ \\
\hline 182.664 & 82228 & (e) & $5 / 2$ & 136974 & (o) & $7 / 2$ & -0.20 & $1.27 \times 10^{9}$ \\
\hline 182.888 & 63246 & (e) & $9 / 2$ & 117925 & (o) & $9 / 2$ & 0.11 & $2.55 \times 10^{9}$ \\
\hline 183.234 & 69346 & (e) & $3 / 2$ & 123921 & (o) & $3 / 2$ & -0.23 & $1.17 \times 10^{9}$ \\
\hline 183.250 & 85506 & (e) & $1 / 2$ & 140076 & (o) & $3 / 2$ & -0.05 & $1.79 \times 10^{9}$ \\
\hline 183.432 & 71686 & (e) & $7 / 2$ & 126203 & (o) & $5 / 2$ & -0.16 & $1.36 \times 10^{9}$ \\
\hline 183.611 & 77409 & (e) & $3 / 2$ & 131872 & & $5 / 2$ & 0.17 & $2.91 \times 10^{9}$ \\
\hline 183.865 & 68139 & (e) & $5 / 2$ & 122 & & $5 / 2$ & -0.10 & $1.59 \times 10^{9}$ \\
\hline 184.016 & 85596 & (e) & $9 / 2$ & 139 & & $11 / 2$ & 0.64 & $8.70 \times 10^{9}$ \\
\hline 184.703 & 85180 & (e) & $3 / 2$ & 1 & & $3 / 2$ & 0.03 & $2.10 \times 10^{9}$ \\
\hline 184.994 & 69346 & (e) & $3 / 2$ & 123 & (o) & $1 / 2$ & -0.23 & $\times 10^{9}$ \\
\hline 185.405 & 73929 & (e) & $5 / 2$ & 127865 & (o) & $3 / 2$ & -0.48 & $6.39 \times 10^{8}$ \\
\hline 185.631 & 68139 & (e) & $5 / 2$ & 122009 & (o) & $3 / 2$ & 0.06 & $2.23 \times 10^{9}$ \\
\hline 185.887 & 73929 & (e) & $5 / 2$ & 127725 & (o) & $7 / 2$ & 0.13 & $2.61 \times 10^{9}$ \\
\hline 186.039 & 111436 & (e) & $1 / 2$ & 165189 & (o) & $1 / 2$ & -0.18 & $1.26 \times 10^{9}$ \\
\hline 186.062 & 76402 & (e) & $5 / 2$ & 130148 & (o) & $5 / 2$ & 0.12 & $2.55 \times 10^{9}$ \\
\hline 186.336 & 85180 & (e) & $3 / 2$ & 138846 & (o) & $5 / 2$ & 0.03 & $2.05 \times 10^{9}$ \\
\hline 186.708 & 71686 & (e) & $7 / 2$ & 125246 & (o) & $9 / 2$ & 0.36 & $4.36 \times 10^{9}$ \\
\hline 186.810 & 82228 & (e) & $5 / 2$ & 135759 & (o) & $5 / 2$ & 0.11 & $2.47 \times 10^{9}$ \\
\hline 186.810 & 76402 & (e) & $5 / 2$ & 129933 & (o) & $3 / 2$ & -0.15 & $1.35 \times 10^{9}$ \\
\hline 187.254 & 71686 & (e) & $7 / 2$ & 125090 & (o) & $5 / 2$ & -0.18 & $1.26 \times 10^{9}$ \\
\hline 187.344 & 65759 & (e) & $7 / 2$ & 119137 & (o) & $5 / 2$ & 0.24 & $3.31 \times 10^{9}$ \\
\hline 187.490 & 80127 & (e) & $3 / 2$ & 133464 & (o) & $1 / 2$ & -0.30 & $9.43 \times 10^{8}$ \\
\hline 188.037 & 69346 & (e) & $3 / 2$ & 122527 & (o) & $5 / 2$ & -0.05 & $1.69 \times 10^{9}$ \\
\hline 188.955 & 68139 & (e) & $5 / 2$ & 121062 & (o) & $7 / 2$ & -0.01 & $1.83 \times 10^{9}$ \\
\hline 189.401 & 73929 & (e) & $5 / 2$ & 126727 & (o) & $7 / 2$ & -0.35 & $8.32 \times 10^{8}$ \\
\hline 189.670 & 82228 & (e) & $5 / 2$ & 134952 & (o) & $3 / 2$ & -0.25 & $1.03 \times 10^{9}$ \\
\hline 191.698 & 65759 & (e) & $7 / 2$ & 117925 & (o) & $9 / 2$ & 0.17 & $2.66 \times 10^{9}$ \\
\hline 191.714 & 63246 & (e) & $9 / 2$ & 115407 & (o) & $7 / 2$ & 0.40 & $4.49 \times 10^{9}$ \\
\hline 192.532 & 71686 & (e) & $7 / 2$ & 123626 & (o) & $7 / 2$ & -0.12 & $1.37 \times 10^{9}$ \\
\hline 193.258 & 80127 & (e) & $3 / 2$ & 131872 & (o) & $5 / 2$ & -0.45 & $6.35 \times 10^{8}$ \\
\hline 193.311 & 77409 & (e) & $3 / 2$ & 129139 & (o) & $1 / 2$ & -0.26 & $9.70 \times 10^{8}$ \\
\hline 194.540 & 82228 & (e) & $5 / 2$ & 133632 & (o) & $7 / 2$ & -0.39 & $7.08 \times 10^{8}$ \\
\hline 194.637 & 85596 & (e) & $9 / 2$ & 136974 & (o) & $7 / 2$ & 0.15 & $2.50 \times 10^{9}$ \\
\hline
\end{tabular}


Table 6. (Continued.)

\begin{tabular}{|c|c|c|c|c|c|c|c|c|}
\hline \multirow{2}{*}{$\begin{array}{l}\text { Wavelength } \\
(\mathrm{nm})\end{array}$} & \multicolumn{3}{|c|}{ Lower level $^{b}$} & \multicolumn{3}{|c|}{ Upper level $^{\mathrm{b}}$} & \multirow[b]{2}{*}{$\log g f^{\mathrm{c}}$} & \multirow{2}{*}{$\begin{array}{l}g A^{\mathrm{c}} \\
\left(\mathrm{s}^{-1}\right)\end{array}$} \\
\hline & $E\left(\mathrm{~cm}^{-1}\right)$ & Parity & $J$ & $E\left(\mathrm{~cm}^{-1}\right)$ & Parity & $J$ & & \\
\hline 194.846 & 76402 & (e) & $5 / 2$ & 127725 & (o) & $7 / 2$ & -0.29 & $9.06 \times 10^{8}$ \\
\hline 195.278 & 85596 & (e) & $9 / 2$ & 136805 & (o) & $9 / 2$ & -0.43 & $6.53 \times 10^{8}$ \\
\hline 195.768 & 85724 & (e) & $7 / 2$ & 136805 & (o) & $9 / 2$ & 0.48 & $5.37 \times 10^{9}$ \\
\hline 197.598 & 71686 & (e) & $7 / 2$ & 122294 & (o) & $9 / 2$ & -0.29 & $8.81 \times 10^{8}$ \\
\hline 197.711 & 85180 & (e) & $3 / 2$ & 135759 & (o) & $5 / 2$ & -0.30 & $8.56 \times 10^{8}$ \\
\hline 199.968 & 73929 & (e) & $5 / 2$ & 123921 & (o) & $3 / 2$ & -0.25 & $9.36 \times 10^{8}$ \\
\hline 216.204 & 71686 & (e) & $7 / 2$ & 117925 & (o) & $9 / 2$ & -0.23 & $8.41 \times 10^{8}$ \\
\hline
\end{tabular}

${ }^{a}$ Wavelength (in vacuum (air) below (above) $200 \mathrm{~nm}$ ) deduced from the experimental

levels.

b Experimental levels from the NIST compilation (Kramida et al 2012).

c This work (HFR+CPOL calculations).

of the scale of transition probabilities deduced in the present work for Rh III, Pd III and Ag III.

\section{Available atomic data in Rh III, Pd III and Ag III}

According to the most recent NIST compilation (Kramida et al 2012), 196 energy levels are known in Rh III. These were taken from the work of Iglesias (1966) who corrected and extended the previous analysis carried out by Catalan et al (1955) and used in Moore's tables (1971). All the experimentally determined levels in Rh III belong to the $4 d^{7}$, $4 d^{6} 5 s$ and $4 d^{6} 5 p$ configurations.

In the case of Pd III, 177 energy levels are listed in the NIST compilation. These were taken from the works due to Shenstone (1963) and Barakat et al (1985). In the former study, the Pd III spectrum was observed from 68.8 to $299.1 \mathrm{~nm}$ allowing the author to identify 917 lines as combinations of 57 even energy levels with 111 of odd parity. With the aid of theoretical predictions, spectral terms from $4 d^{8}, 4 d^{7} 5 \mathrm{~s}$, $4 d^{7} 6 s, 4 d^{7} 5 p$ and $4 d^{6} 5 s 5 p$ configurations were designated. In Barakat et al's work, the spectrum of palladium was photographed in the wavelength region $45-230 \mathrm{~nm}$ using a $6.650 \mathrm{~m}$ normal incidence vacuum spectrograph and a sliding spark as light source. The missing levels in $4 \mathrm{~d}^{8}, 4 \mathrm{~d}^{7} 5 \mathrm{~s}$ and $4 d^{7} 5 p$ configurations were determined and 326 lines which were not recorded in a previous analysis were added to the classified lines in this spectrum.

For Ag III, the 64 levels reported in the NIST compilation belong to the $4 d^{9}, 4 d^{8} 5 s$ and $4 d^{8} 5 p$ configurations. These were taken from the work of Benschop et al (1975) who measured the silver spectrum between 40 and $230 \mathrm{~nm}$ using different spectrographs including the $10.7 \mathrm{~m}$ normal incidence vacuum spectrograph at the NBS Laboratory in Washington DC. In this work, some levels published earlier by Gilbert (1935) were rejected and all the missing levels in the $4 d^{8} 5 s$ and $4 d^{8} 5 p$ configurations were identified.

On the theoretical side, Shadmi (1966) reported a systematic treatment of the configurations $4 d^{k}+4 d^{k-1} 5 \mathrm{~s}$ in doubly ionized atoms of the palladium group, including Rh III, Pd III and Ag III based on the Slater approximation with several improvements. Let us also mention that the Slater-Racah parametric method combined with generalized least-squares fits of the energy parameters was used by Wyart et al (1993) to study the $(4 d+5 s)^{8}$ mixed configurations along the $\mathrm{Ru}$ isoelectronic sequence from $\mathrm{Rh}$ II to $\mathrm{Sb} \mathrm{VIII}$, therefore including Pd III.

To our knowledge, no radiative rates have been published so far for doubly ionized rhodium, palladium and silver.

\section{Atomic structure calculations}

The HFR method developed by Cowan (1981) was used to get the atomic orbitals of the three ions considered and the following configurations were retained in the configuration interaction expansions :

For Rh III:

$4 d^{7}+4 d^{6} 5 s+4 d^{6} 6 s+4 d^{6} 5 d+4 d^{6} 6 d+4 d^{5} 5 s^{2}+4 d^{5} 5 p^{2}+$ $4 d^{5} 5 d^{2}+4 d^{5} 5 s 6 s$ (even parity) and $4 d^{6} 5 p+4 d^{6} 6 p+4 d^{6} 4 f+$ $4 d^{6} 5 f+4 d^{5} 5 s 5 p+4 d^{5} 5 s 6 p+4 d^{5} 5 p 6 s$ (odd parity).

For Pd III:

$4 d^{8}+4 d^{7} 5 s+4 d^{7} 6 s+4 d^{7} 5 d+4 d^{7} 6 d+4 d^{6} 5 s^{2}+4 d^{6} 5 p^{2}$ $+4 d^{6} 5 d^{2}+4 d^{6} 5 s 6 s+4 d^{6} 5 s 5 d+4 d^{6} 5 s 6 d$ (even parity) and $4 d^{7} 5 p+4 d^{7} 6 p+4 d^{7} 4 f+4 d^{7} 5 f+4 d^{6} 5 s 5 p+4 d^{6} 5 s 6 p+$ $4 d^{6} 5 p 5 d+4 d^{6} 5 p 6 s$ (odd parity).

For Ag III:

$4 d^{9}+4 d^{8} 5 s+4 d^{8} 6 s+4 d^{8} 5 d+4 d^{8} 6 d+4 d^{7} 5 s^{2}+4 d^{7} 5 p^{2}$ $+4 d^{7} 5 d^{2}+4 d^{7} 5 s 6 s+4 d^{7} 5 s 5 d+4 d^{7} 5 s 6 d$ (even parity) and $4 d^{8} 5 p+4 d^{8} 6 p+4 d^{8} 4 f+4 d^{8} 5 f+4 d^{7} 5 s 5 p+4 d^{7} 5 s 6 p+$ $4 d^{7} 5 p 5 d+4 d^{7} 5 p 6 s$ (odd parity).

Besides these intravalence correlations, core-valence interactions were taken into account using a core-polarization model potential and a correction to the electric dipole transition operator, both depending on two parameters (the dipole polarizability of the ionic core, $\alpha_{\mathrm{d}}$, and the cut-off radius, $r_{\mathrm{c}}$ ). This technique has been implemented in Cowan's codes and described in details elsewhere (see e.g. Quinet et al 1999). The dipole polarizability values used in our calculations were taken from Fraga et al (1976), i.e. $\alpha_{\mathrm{d}}=$ 3.31, 3.17 and $3.04 a_{0}^{3}$ corresponding to $\mathrm{Rh} \mathrm{V}, \mathrm{Pd} \mathrm{V}$ and $\mathrm{Ag} \mathrm{V}$ cores, respectively. For the cut-off radius, we adopted the values $r_{\mathrm{c}}=1.43,1.36$ and $1.30 a_{0}$ which correspond to the HFR mean values of $r$ for the outermost $4 \mathrm{~d}$ orbital of the ionic core in Rh III, Pd III and Ag III, respectively.

For each ion, the HFR+CPOL method described above was then combined to a semi-empirical adjustment of radial energy parameters in order to minimize the differences between calculated Hamiltonian eigenvalues and available experimental energy levels. For Rh III, all the levels tabulated 
in the NIST compilation (Kramida et al 2012) were used to fit the radial parameters corresponding to the $4 d^{7}, 4 d^{6} 5 s$ and $4 d^{6} 5 p$ configurations if we except the level situated at $133995.8 \mathrm{~cm}^{-1}(J=7 / 2$, odd parity $)$ for which no correspondence was found in our calculations. In the case of Pd III, the fitting process was carried out with all the levels compiled at NIST for the $4 d^{8}, 4 d^{7} 5 s$ and $4 d^{7} 5 p$, i.e. up to $163470.1 \mathrm{~cm}^{-1}$. The experimental levels above that limit were not included in the fit because many of them are given with an uncertain energy value or an unknown electronic configuration. For Ag III, all the levels listed by the NIST were considered in the semi-empirical fitting. They were used to optimize the radial parameters in $4 d^{9}, 4 d^{8} 5 s$ and $4 d^{8} 5 p$ configurations. For the three ions, the Slater integrals not optimized in the fitting process were scaled down by a factor 0.90 as suggested by Cowan (1981).

Energy levels obtained in the present work are compared to available experimental values in tables 1-3 (given as supplementary data (available from stacks.iop.org/PhysScr/88/065302/mmedia)) for Rh III, Pd III and Ag III, respectively. We can observe an excellent agreement between both sets of data, the average differences being found to be equal to $28 \mathrm{~cm}^{-1}$ (even parity) and $84 \mathrm{~cm}^{-1}$ (odd parity) for Rh III, $80 \mathrm{~cm}^{-1}$ (even parity) and $68 \mathrm{~cm}^{-1}$ (odd parity) for Pd III and $117 \mathrm{~cm}^{-1}$ (even parity) and $66 \mathrm{~cm}^{-1}$ (odd parity) in the case of $\mathrm{Ag}$ III. Note that, for $\mathrm{Rh}$ III, the energies of nine $4 d^{6} 5 p$ levels have been estimated for the first time at $122442 \mathrm{~cm}^{-1}(J=3 / 2), 124589 \mathrm{~cm}^{-1}$ $(J=7 / 2), 129557 \mathrm{~cm}^{-1}(J=5 / 2), 131606 \mathrm{~cm}^{-1}(J=1 / 2)$, $133998 \mathrm{~cm}^{-1} \quad(J=5 / 2), \quad 134621 \mathrm{~cm}^{-1} \quad(J=1 / 2)$, $134918 \mathrm{~cm}^{-1} \quad(J=3 / 2), \quad 135589 \mathrm{~cm}^{-1} \quad(J=3 / 2) \quad$ and $135719 \mathrm{~cm}^{-1}(J=5 / 2)$. As also shown in those tables, the level mixing is much stronger in the $4 \mathrm{~d}^{k-1} 5 \mathrm{p}$ odd-parity configurations than in $4 \mathrm{~d}^{k}$ and $4 \mathrm{~d}^{k-1} 5 \mathrm{~s}$ even-parity configurations, the average $L S$ purities being found equal to $87 \%\left(4 d^{7}\right), 74 \%\left(4 d^{6} 5 s\right)$ and $45 \%\left(4 d^{6} 5 p\right)$ in Rh III, $87 \%$ $\left(4 d^{8}\right), 76 \%\left(4 d^{7} 5 s\right)$ and $52 \%\left(4 d^{7} 5 p\right)$ in Pd III and $99 \%\left(4 d^{9}\right)$, $82 \%\left(4 d^{8} 5 \mathrm{~s}\right)$ and $58 \%\left(4 d^{8} 5 \mathrm{p}\right)$ in $\mathrm{Ag}$ III.

\section{Oscillator strengths and transition probabilities}

Computed oscillator strengths and transition probabilities for spectral lines of $\mathrm{Rh}$ III, Pd III and Ag III are given in tables 4-6, respectively. All these lines, appearing in the ultraviolet region from 70 to $250 \mathrm{~nm}$, correspond to transitions of the type $4 \mathrm{~d}^{k}-4 \mathrm{~d}^{k-1} 5 \mathrm{p}$ and $4 \mathrm{~d}^{k-1} 5 \mathrm{~s}-4 \mathrm{~d}^{k-1} 5 \mathrm{p}$ with $k=7,8,9$ for Rh III, Pd III and Ag III, respectively. Due to space limitations, spectroscopic designations of levels are not included in the tables. Such designations are reported in tables 1-3 given as supplementary data (available from stacks.iop.org/PhysScr/88/065302/mmedia). Note that the results presented in the tables are limited to the most intense transitions characterized by $\log g f$-values greater than -0.5 . Extensive tables containing the whole sets of data obtained in the present work (with radiative parameters for about $2150 \mathrm{Rh}$ III lines ranging from 76 to $519 \mathrm{~nm}, 2120 \mathrm{Pd} \mathrm{III}$ lines ranging from 61 to $975 \mathrm{~nm}$ and $440 \mathrm{Ag}$ III lines ranging from 59 to $801 \mathrm{~nm}$ ) can be obtained as supplementary files upon request to the authors.

Unfortunately, no experimental neither previous theoretical radiative rates in these three ions are available for comparison. Nevertheless, an argument for assessing the reliability of the present results can be obtained from isoelectronic comparisons, in particular from results published recently by Palmeri et al (2009) in $\mathrm{Ru}$ II (isoelectronic of Rh III) and by Quinet et al (2012) in $\mathrm{Rh}$ II (isoelectronic of $\mathrm{Pd}$ III), the $\mathrm{HFR}+\mathrm{CPOL}$ models adopted in the present work being the same as those chosen for these ions. More precisely, in these two latter papers, radiative lifetimes of 23 states in $\mathrm{Ru}$ II and 17 states in $\mathrm{Rh}$ II were accurately measured by means of the time-resolved laser-induced fluorescence (TR-LIF) technique. Free singly ionized ruthenium or rhodium ions were obtained in a laser-produced plasma. A tunable laser, with $1 \mathrm{~ns}$ duration pulse, was used to selectively excite the $\mathrm{Ru}^{+}$and $\mathrm{Rh}^{+}$ions. The lifetime values were evaluated from the transient LIF signals recorded by a fast detection system. In both ions, a comparison of the HFR+CPOL and the experimental lifetimes showed that the calculated values were in excellent agreement (within a few per cent) with the measurements. A similar accuracy can thus be expected for the decay rates computed for the doubly charged ions considered in the present work, at least for the most intense transitions.

\section{Acknowledgments}

Financial support from the Belgian FRS-FNRS is acknowledged. WZ, PP and PQ are respectively Short term Foreign Postdoctoral Fellow, Research Associate and Research Director of this organization.

\section{References}

Barakat M M, van Kleef Th A M and Raassen A J J 1985 Physica B+C 132111

Benschop H, Joshi Y N and van Kleef Th A M 1975 Can. J. Phys. 53498

Biémont E et al 2005 J. Electron Spectrosc. Relat. Phenom. 144-147 27

Biémont E et al 2011 Mon. Not. R. Astron. Soc. 4143350

Campos J et al 2005 Mon. Not. R. Astron. Soc. 363905

Catalan M A, Sales M and Shenstone A G 1955 unpublished

Cowan R D 1981 The Theory of Atomic Structure and Spectra (Berkeley, CA: University of California Press)

Fivet V et al 2009 Mon. Not. R. Astron. Soc. 3962124

Fraga S, Karwowski J and Saxena K M S 1976 Handbook of Atomic Data (Amsterdam: Elsevier)

Gilbert W P 1935 Phys. Rev. 48338

Hansen C J et al 2012 Astron. Astrophys. 545 A31

Hartman $\mathrm{H}$ et al 2010 Phys. Rev. A 82052512

Iglesias L 1966 Can. J. Phys. 44895

Jiang L Y et al 2012 Eur. Phys. J. D 66176

Jorissen A 2004 Phys. Scr. T112 73

Kramida A, Ralchenko Yu, Reader J (NIST ASD Team) 2012 NIST Atomic Spectra Database http://physics.nist.gov/asd

Lundberg H et al 2010 J. Phys. B: At. Mol. Opt. Phys. 43085004

Malcheva G et al 2006 Mon. Not. R. Astron. Soc. 367754

Malcheva G et al 2011 Mon. Not. R. Astron. Soc. 4121823

Mashonkina L 2009 Phys. Scr. T134 014004

Moore C E 1971 Atomic Energy Levels NSRDS 35 III p 34

Nilsson $\mathrm{H}$ et al 2010 Astron. Astrophys. 511 A16

Palmeri P et al 2007 Mon. Not. R. Astron. Soc. 37463

Palmeri P et al 2009 J. Phys. B: At. Mol. Opt. Phys. 42165005

Quinet P 2002 J. Phys. B: At. Mol. Opt. Phys. 3519 
Quinet P et al 2011 J. Electron Spectrosc. Relat. Phenom. 184174 Quinet P et al 2012 Astron. Astrophys. 537 A74

Quinet P, Palmeri P, Biémont E, McCurdy M M, Rieger G,

Pinnington E H, Wickliffe M E and Lawler J E 1999 Mon. Not. R. Astron. Soc. 307934

Shadmi Y 1966 J. Res. Natl Bur. Stand. A70 435

Shenstone A G 1963 J. Res. Natl Bur. Stand. A67 87

Wallerstein G et al 1997 Rev. Mod. Phys. 69995
Wyart J-F, Raassen A J J, Uylings P H M and Joshi Y N 1993 Phys. Scr. T47 59

Xu H L et al 2006 Astron. Astrophys. 452357

Zhang W et al 2009 Eur. Phys. J. D 551

Zhang W et al 2010 J. Phys. B: At. Mol. Opt. Phys. 43205005

Zhang W, Palmeri P, Quinet P and Biémont E 2013 Astron. Astrophys. 551 A136

Zhang Y et al 2008 Phys. Rev. A 78022505 